On the diffraction of acoustic waves by a quarter-plane

Assier, Raphael and Peake, Nigel

2012

MIMS EPrint: 2014.60

Manchester Institute for Mathematical Sciences

School of Mathematics

The University of Manchester

\footnotetext{
Reports available from: http://eprints.maths.manchester.ac.uk/

And by contacting: The MIMS Secretary

School of Mathematics

The University of Manchester

Manchester, M13 9PL, UK
} 


\title{
On the diffraction of acoustic waves by a quarter-plane
}

\author{
R.C. Assier ${ }^{a, *}$, N. Peake ${ }^{a}$ \\ ${ }^{a}$ DAMTP, University of Cambridge, Wilberforce Road, Cambridge CB3 OWA,UK
}

\begin{abstract}
This paper follows the work of A.V. Shanin on diffraction by an ideal quarter-plane. Shanin's theory, based on embedding formulae, the acoustic uniqueness theorem and spherical edge Green's functions, leads to three modified Smyshlyaev formulae, which partially solve the far-field problem of scattering of an incident plane wave by a quarter-plane in the Dirichlet case. In this paper, we present similar formulae in the Neumann case, and describe a numerical method allowing a fast computation of the diffraction coefficient using Shanin's third modified Smyshlyaev formula. The method requires knowledge of the eigenvalues of the Laplace-Beltrami operator on the unit sphere with a cut, and we also describe a way of computing these eigenvalues. Numerical results are given for different directions of incident plane wave in the Dirichlet and the Neumann cases, emphasising the superiority of the third modified Smyshlyaev formula over the other two.
\end{abstract}

Keywords: quarter-plane, acoustics, diffraction, far-field

\section{Introduction}

The diffraction of acoustic waves by an ideal quarter-plane is a complex problem, which has so far proved insoluble via classical techniques. This problem is an important canonical model for the Geometrical Theory of Diffraction (GTD) due to Keller [1]. Following the GTD, further work has been done by Kraus and Levine [2], Satterwhite [3] and Hansen [4], by considering the quarter-plane as a degenerated elliptic cone and expressing the field as a spherical wave multipole series. However, these series are poorly convergent when the source and the observer are located far from the vertex of the cone. A more extensive review has been undertaken by Blume in [5]. In the same paper [5], Blume proposes a method to accelerate the convergence of these series and obtains some numerical results. In 1961, Radlow [6] claimed to have found an analytic solution of the acoustic quarter-plane problem using the Wiener-Hopf method. However, this solution has long been suspected to be wrong (see Meister [7]), and indeed it has recently been disproved by Albani [8]. A different way to attack the quarter-plane problem has been introduced by Smyshlyaev [9], followed by some work by Babich et al. [10], but in this case the solution is still difficult to evaluate numerically. Despite this difficulty, Babich et al [11] describe a numerical method based on the Abel-Poissontype summation method and a boundary integral equation that gives the diffraction coefficient for smooth convex cones in the non-singular directions. This method has also been successfully implemented by Bonner et al [12] for more complicated geometries. However, Shanin [13, 14], following Smyshlyaev's work, presents a new analytical/numerical method, which partially solves the acoustic quarter-plane problem in the Dirichlet case. The main advantage of this method compared to the one mentioned previously is that in this case the formulae giving the diffraction coefficient are "naturally convergent" in the sense that they don't require a special treatment to regularise or accelerate their convergence. This method is mainly based on the theory of embedding formulae, introduced by Williams [15] and developed by Craster, Shanin and others [16, 17, 18].

\footnotetext{
*Corresponding author, Tel: +44 01223766 349, Fax: +44 (0) 1223765900

Email addresses: rca32@cam.ac.uk (R.C. Assier), n.peake@damtp.cam.ac.uk (N. Peake)
} 
It also relies on the acoustic uniqueness theorem for infinite scatterers, which has been discussed in detail by Jones [19] and Miranker [20]. In the present paper, we will extend Shanin's theory to the Neumann case, and present numerical results in both cases over a broader range of observer locations (using the third modified Smyshlyaev formula) than was possible with the numerical methods presented in previous papers (using the first two modified Smyshlyaev formulae only). In Section 2 we shall give a mathematical formulation of the problem. In Section 3 this problem will be rewritten as a spherical eigenvalue problem and a method to obtain the eigenvalues numerically will be described. In Section 4, integral formulae describing the solution, the modified Smyshlyaev formulae, shall be derived in the Dirichlet and the Neumann cases and a method to compute their integrands will be presented. Section 5 will describe an efficient way of computing the far-field directivity, and numerical results and physical interpretation shall be given in Section 6 .

\section{Notations and problem formulation}

For brevity, we will treat the Dirichlet and Neumann cases simultaneously. The convention used throughout is that the grey upper cell concerns the Dirichlet case and the lower white one concerns the Neumann case, and that the upper index ${ }^{d, n}$ concerns the Dirichlet/Neumann cases respectively. Let us introduce the functions $\mathfrak{T}^{d, n}, \mathfrak{d}^{d, n}$ and $\mathfrak{S}_{\alpha}^{d, n}$ defined by

$$
\mathfrak{T}^{d, n}=\left[\begin{array}{l}
\sin \\
\cos
\end{array}\right], \quad \mathfrak{d}^{d, n}=\left[\begin{array}{c}
1 \\
-1
\end{array}\right], \quad \mathfrak{S}_{\alpha}^{d, n}(z)=\left[\begin{array}{c}
\delta(z-\alpha) \\
\frac{\delta\left(z-0^{+}\right)-\delta\left(z-0^{-}\right)}{2}
\end{array}\right],
$$

where $\delta$ is the usual Dirac delta function. We shall consider the time-harmonic scalar Dirichlet/Neumann diffraction problem, sending an incident plane wave with velocity potential of the form $u_{\text {in }} e^{-i \Omega t}$, where $u_{\text {in }}=e^{-i\left(k_{x} x+k_{y} y+k_{z} z\right)}$, onto a quarter plane, as shown in Figure 1. The direction of the incident wave can be represented by the unit vector $\omega_{0}=\left(k_{x}, k_{y}, k_{z}\right) / k_{0}$. It is also possible to describe $\omega_{0}$ by its spherical coordinates $\left(\theta_{0}, \varphi_{0}\right)$ as can be seen in Figure 2. In what follows the time dependence will be suppressed. According to Keller's GTD [1], the total velocity potential field $u_{\text {tot }}^{d, n}$ has a quite complex structure, as illustrated in Figure 1. It consists of an incident plane wave $u_{\text {in }}$, a reflected plane wave $u_{\text {re }}^{d, n}$ present in the geometric scattered region and a spherical wave coming from the vertex $u_{\mathrm{sp}}^{d, n}$. Inside the geometric scattered region, $u_{\mathrm{re}}^{d, n}$ is equal to $-\mathfrak{d}^{d, n} e^{-i\left(k_{x} x+k_{y} y-k_{z} z\right)}$. Outside this region, $u_{\mathrm{re}}^{d, n}$ is equal to zero. There is also a set of conical/cylindrical waves radiated from each edge, denoted $u_{\mathrm{co} 1}^{d, n}$ and $u_{\mathrm{co} 2}^{d, n}$, and in some cases (see Table 1), a set of secondary radiated conical waves radiated from the edges denoted $u_{\mathrm{co} 21}^{d, n}$ and $u_{\mathrm{co} 12}^{d, n}$. Let us introduce the scattered field $u_{\mathrm{sc}}^{d, n}$, such that $u_{\mathrm{tot}}^{d, n}=u_{\mathrm{in}}+u_{\mathrm{re}}^{d, n}+u_{\mathrm{sc}}^{d, n}$. Hence we can write

$$
u_{\mathrm{sc}}^{d, n}=u_{\mathrm{sp}}^{d, n}+u_{\mathrm{co} 1}^{d, n}+u_{\mathrm{co} 2}^{d, n}+u_{\mathrm{co} 21}^{d, n}+u_{\mathrm{co} 12}^{d, n}
$$

\begin{tabular}{|c|c|c|c|c|}
\hline$\varphi_{0}$ domain & {$\left[0, \frac{\pi}{2}\right]$} & {$\left[\frac{\pi}{2}, \pi\right]$} & {$\left[\pi, \frac{3 \pi}{2}\right]$} & {$\left[\frac{3 \pi}{2}, 2 \pi\right]$} \\
\hline$u_{\mathrm{co} 12}^{d, n}$ & $\checkmark$ & $\checkmark$ & $\mathrm{O}$ & $\mathrm{O}$ \\
\hline$u_{\mathrm{co} 21}^{d, n}$ & $\checkmark$ & $\mathrm{O}$ & $\mathrm{O}$ & $\checkmark$ \\
\hline
\end{tabular}

Table 1: Occurrence of the secondary radiated conical waves, the $\checkmark$ means occurrence and the O means absence 


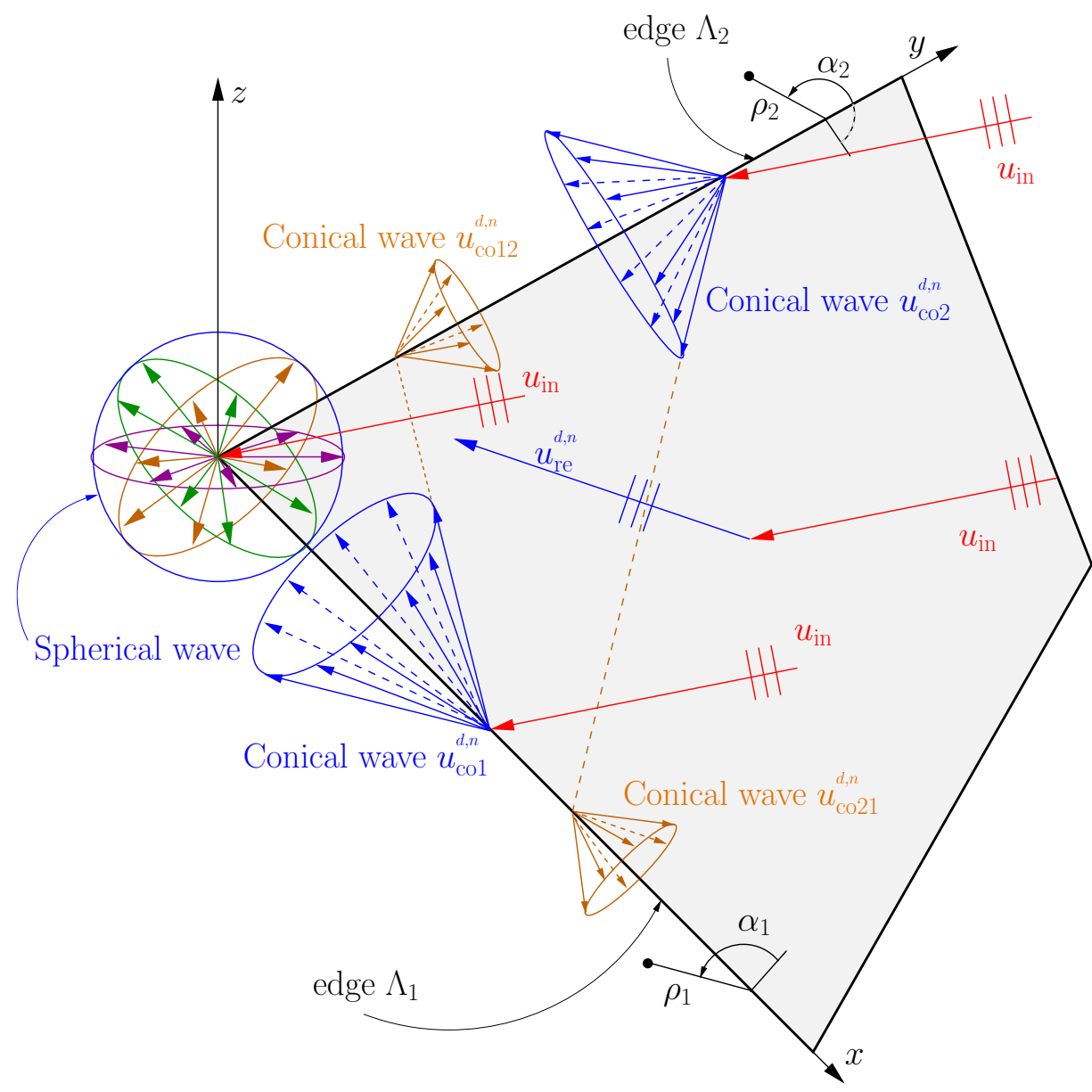

Figure 1: Illustration of the structure of the total field for $\varphi_{0} \in\left[0, \frac{\pi}{2}\right]$

All these fields satisfy the Helmholtz equation $\Delta u+k_{0}^{2} u=0$, where $k_{0}=\sqrt{k_{x}^{2}+k_{y}^{2}+k_{z}^{2}}$ is the wave number of $u_{\text {in }}$, subject to the following conditions:

- The boundary conditions $u_{\mathrm{sc}}^{d}=0$ and $\frac{\partial u_{\mathrm{sc}}^{n}}{\partial z}=0$ on both surfaces of the quarter-plane.

- The edge or Meixner conditions $u_{\mathrm{sc}}^{d, n} \sim \rho_{1,2}^{1 / 2} \mathfrak{T}^{d, n}\left(\frac{\alpha_{1,2}}{2}\right)$ near the edge $\Lambda_{1,2}$ (coordinates described on Figure 1). These conditions follow from the fact that very close to the edges the quarter-plane looks exactly like a half-plane. These conditions may also be interpreted as preventing any source from being located on the edges.

- The vertex condition $\nabla u_{\mathrm{sc}}^{d, n}=o\left(r^{-3 / 2}\right)$ as $r \rightarrow 0, r$ being the distance from the vertex of the quarterplane. This condition follows from the fact that the energy density should be integrable at the vertex.

The spherical behaviour of $u_{\mathrm{sp}}^{d, n}$ in the far-field (i.e. as $k_{0} r \rightarrow \infty$ ) can be expressed by writing

$$
u_{\mathrm{sp}}^{d, n}(\omega, r) \sim 2 \pi \frac{e^{i k_{0} r}}{k_{0} r} f^{d, n}\left(\omega, \omega_{0}\right),
$$

where $\omega_{0}=\left(k_{x}, k_{y}, k_{z}\right) / k_{0}$ is the unit vector of incidence and $\omega$ is the unit vector of observation, as shown in Figure 2. It is expected for the diffraction coefficient $f^{d, n}\left(\omega, \omega_{0}\right)$ to become singular at the geometrical 
optics boundaries, that in our case correspond with the directions of the radiated and secondary radiated cones described previously. It is possible to derive uniformly valid far-field expressions in some problems (e.g. [21, 22] for radiation from skewed cylinder or [23, 24] for radiation from cones), but that will not be considered further here. A formula has been found for the diffraction coefficient $f^{d, n}\left(\omega, \omega_{0}\right)$ by Smyshlyaev $[9]$, which states that

$$
f^{d, n}\left(\omega, \omega_{0}\right)=\frac{i}{\pi} \int_{\gamma} e^{-i \pi \nu} g^{d, n}\left(\omega, \omega_{0}, \nu\right) \nu d \nu
$$

where $g^{d, n}$ is the Green's function of the spherical problem, $\nu$ is the separation constant of the spherical problem (see Section 4) and $\gamma$ is an integration contour which will be defined later. However, as mentioned in the introduction, expression (2) is difficult to use for practical computations. It is indeed only "naturally convergent" for a very restricted range of ordered pairs $\left(\omega, \omega_{0}\right)$. On this restricted range of ordered pairs, (2) can indeed be written as an exponentially convergent integral as shown in [9] and implemented in [10]. In the particular case of an axi-symmetric problem, Babich et al showed in [10] that, using earlier ideas of Nikolaev [25], (2) could be modified into an exponentially convergent integral everywhere. In a more recent paper, Shanin [13] improved (2) by introducing three modified Smyshlyaev formulae in the Dirichlet case. The main difference between the three formulae is the range of ordered pairs $\left(\omega, \omega_{0}\right)$ for which the integrand is exponentially decaying along the imaginary axis (allowing contour deformation and efficient numerical evaluation as shown in Section 5). Shanin and co-worker have considered only the first and second modified Smyshlyaev formulae. In this paper, we will mainly focus on the third modified Smyshlyaev formula, which is the one with the greatest range of validity.

The vertex of the quarter-plane is taken as the origin, and the usual Cartesian coordinates $(x, y, z)$ and spherical coordinates $(r, \theta, \varphi)$ are shown in Figure 2. The spherical coordinates may also be written as $(\omega, r)$ where $\omega$ corresponds to the intersection between the vector and the unit sphere. $\omega$ can be described by the ordered pair $(\xi, \eta)$, which corresponds to the Cartesian coordinates of its projection on the equatorial plane $z=0$, as shown in Figure 2. It is trivial to show that $\xi=\sin (\theta) \cos (\varphi)$ and $\eta=\sin (\theta) \sin (\varphi)$.

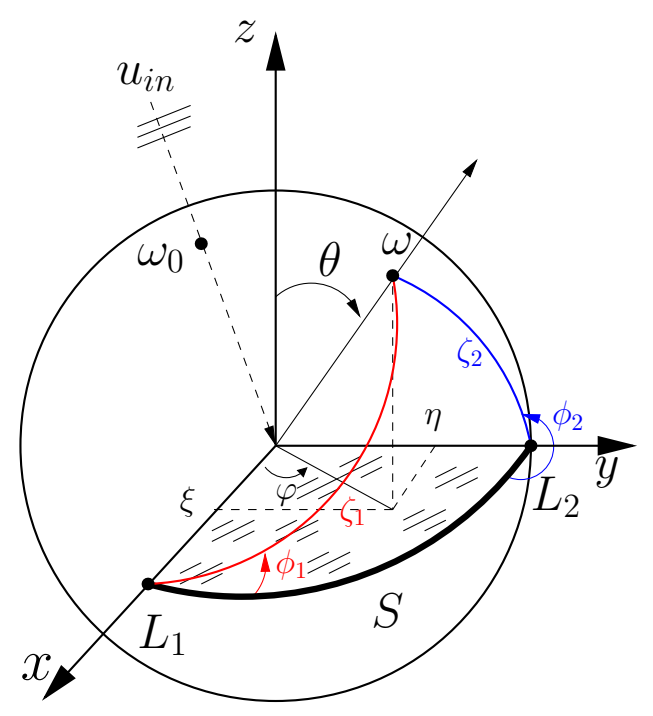

Figure 2: Coordinates on a sphere

Local cylindrical coordinates are used very close to the edges of the quarter-plane, denoted $\Lambda_{1}(x$ axis) and $\Lambda_{2}$ ( $y$ axis) respectively. For example, for $\Lambda_{1}$ the local cylindrical coordinates are $\left(x, \rho_{1}, \alpha_{1}\right)$, as shown in Figure 1. We have $x=\rho_{2} \cos \left(\alpha_{2}\right), y=\rho_{1} \cos \left(\alpha_{1}\right)$ and $z=\rho_{1,2} \sin \left(\alpha_{1,2}\right)$. Local coordinates on the sphere 
$\left(\zeta_{1,2}, \phi_{1,2}\right)$ are used to determine the position of a point $\omega$ on the unit sphere relative to the cut $S$, which corresponds to the cross-section between the unit sphere and the quarter-plane. Let us denote the two extremities of $S$ as $L_{1}$ and $L_{2}$. As can be seen in Figure 2, $\zeta_{1,2}$ correspond to the length of the geodesics between $L_{1,2}$ and $\omega$. The angles $\phi_{1,2}$ are the spherical angles between the equator and these geodesics. These local coordinates can also be used as global coordinates on the unit sphere, and can be linked with the usual spherical coordinates by

$$
\begin{array}{cl}
\cos \left(\zeta_{1}\right)=\sin (\theta) \cos (\varphi) \quad, \quad \cos \left(\zeta_{2}\right)=\sin (\theta) \sin (\varphi) \\
\tan \left(\phi_{1}\right)=\frac{\cos (\theta)}{\sin (\theta) \sin (\varphi)} \quad, \quad \tan \left(\phi_{2}\right)=-\frac{\cos (\theta)}{\sin (\theta) \cos (\varphi)}
\end{array}
$$

Note that the minus sign in (4) comes from the fact that $\phi_{2}$ is defined as the complement of the acute angle. Note that we also have $\xi=\cos \left(\zeta_{1}\right)$ and $\eta=\cos \left(\zeta_{2}\right)$.

\section{On the equivalent spherical problem}

Let us now operate the change of variable $u^{d, n}(r, \theta, \varphi)=R^{d, n}(r) V^{d, n}(\theta, \varphi)$. A particular solution of the radial part is $R^{d, n}(r)=\frac{A^{d, n}}{\sqrt{k_{0} r}} J_{\nu}\left(k_{0} r\right)$, while the spherical part $V^{d, n}$ should satisfy the Laplace-Beltrami problem $\left(P_{1}^{\prime}\right)$, where

$$
\left(P_{1}^{\prime}\right)=\left\{\begin{array}{c}
\left(\tilde{\Delta}+\nu^{2}-\frac{1}{4}\right) V^{d, n}(\omega, \nu)=0 \\
V^{d}=0, \quad \frac{\partial V^{n}}{\partial n}=0 \quad \text { on } S \\
V^{d, n} \sim \zeta_{1,2}^{1 / 2} \mathfrak{T}^{d, n}\left(\frac{\phi_{1,2}}{2}\right) \text { near } L_{1,2}
\end{array}\right.
$$

and $\tilde{\Delta}$ is the Laplace-Beltrami operator defined by $\tilde{\Delta}=\frac{1}{\sin (\theta)} \frac{\partial}{\partial \theta}\left(\sin \theta \frac{\partial}{\partial \theta}\right)+\frac{1}{\sin ^{2} \theta} \frac{\partial^{2}}{\partial \varphi^{2}}$. Let us consider only the symmetric/antisymmetric eigenfunctions of the Dirichlet/Neumann problem (5), denoted as $\phi_{j}^{d, n}(\omega)$ with corresponding eigenvalues $\nu_{j}^{d, n}$, so that we have $\phi_{j}^{d}(\theta, \varphi)=\phi_{j}^{d}(\pi-\theta, \varphi)$ and $\phi_{j}^{n}(\theta, \varphi)=-\phi_{j}^{n}(\pi-\theta, \varphi)$. As shall be seen in Section 4, knowledge of these eigenvalues is key to a thorough understanding of this problem. Moreover, it can be shown that the behaviour of $u_{\mathrm{sc}}^{d, n}$ near the vertex is given by

$$
u_{\mathrm{sc}}^{d, n} \sim r^{\left(\nu_{1}^{d, n}-1 / 2\right)} \text { as } r \rightarrow 0
$$

In the following subsections we shall present a way of obtaining an accurate numerical estimation of the first few eigenvalues in both the Dirichlet and the Neumann cases. In a report by Jansen and Boersma [26] on the electro-magnetic diffraction by elliptic cones, the first eigenvalues $\nu_{1}^{d, n}$ have been computed and our results agree with theirs up to four decimal places. In this report, Jansen and Boersma used a different method based on Lamé functions that will not be discussed further. Other authors, such as Sleeman et al. [27] and Abawi et al. [28] also have computed these eigenvalues using different methods.

\subsection{The Dirichlet case}

Let us consider the Dirichlet part of problem (5) as a two-dimensional PDE on the domain $\Omega^{d}$ in the $(\varphi, \theta)$ space, as described in Figure 3. 


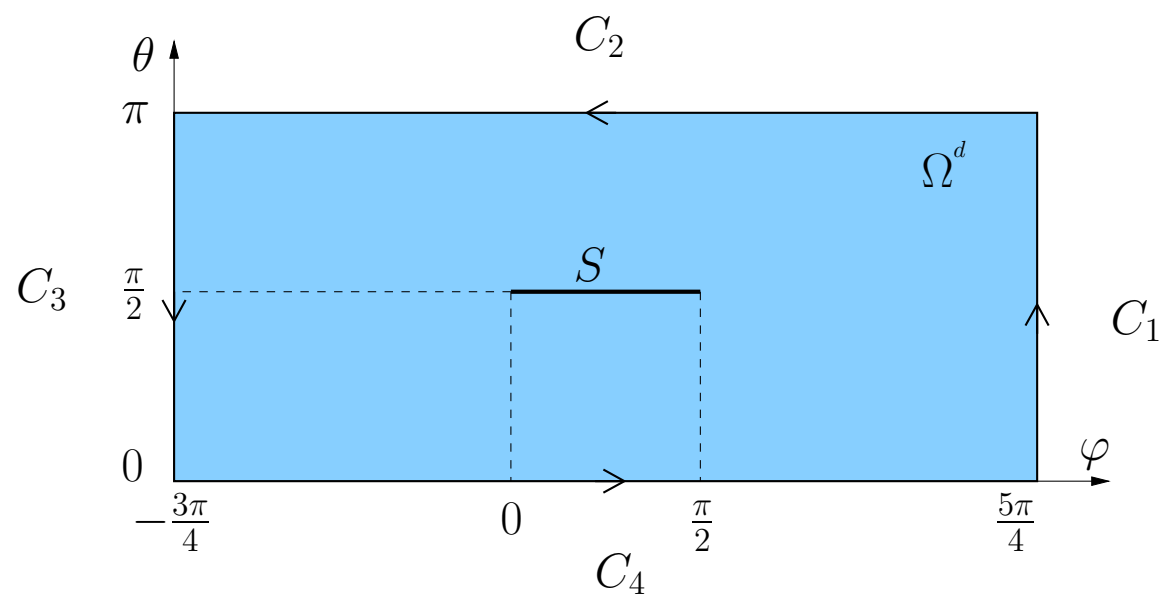

Figure 3: Domain of integration $\Omega^{d}$ in the Dirichlet case

For $\Omega^{d}$ to be equivalent to the unit sphere we need to carefully choose the boundary conditions. On $S$, the Dirichlet boundary conditions $v^{d}(S)=0$ should be satisfied. We want the solutions to be $2 \pi$-periodic in the $\varphi$ direction, therefore we need to impose $v^{d}\left(C_{1}\right)=v^{d}\left(C_{3}\right)$. The boundaries $C_{2}$ and $C_{4}$ correspond to the South and the North pole of the sphere respectively. So the value of $v^{d}$ must be constant on this boundary, but this constant is unknown. Being only interested in eigenvalues which lead to symmetric eigenfunctions, we shall only impose a periodic condition on $C_{2}$ and $C_{4}$ and "select" the appropriate modes detected. A finite element method is used to solve the eigenvalue problem. The domain $\Omega^{d}$ is meshed with a triangular mesh and the finite element space chosen is the space of continuous quadratic functions on the elements of the mesh plus bubble functions. The variational formulation of the problem, (A.4), can be found in Appendix A. The eigenvalue problem is solved using Implicitly Restarted Arnoldi Method (IRAM) using the finite element software Freefem $++[29]$. Figure 4 shows the plot of the first two modes found by the solver, and Table 2 summarises the results obtained for the first six eigenvalues. A mesh analysis has been performed to ensure that these results have a precision of order $10^{-4}$.
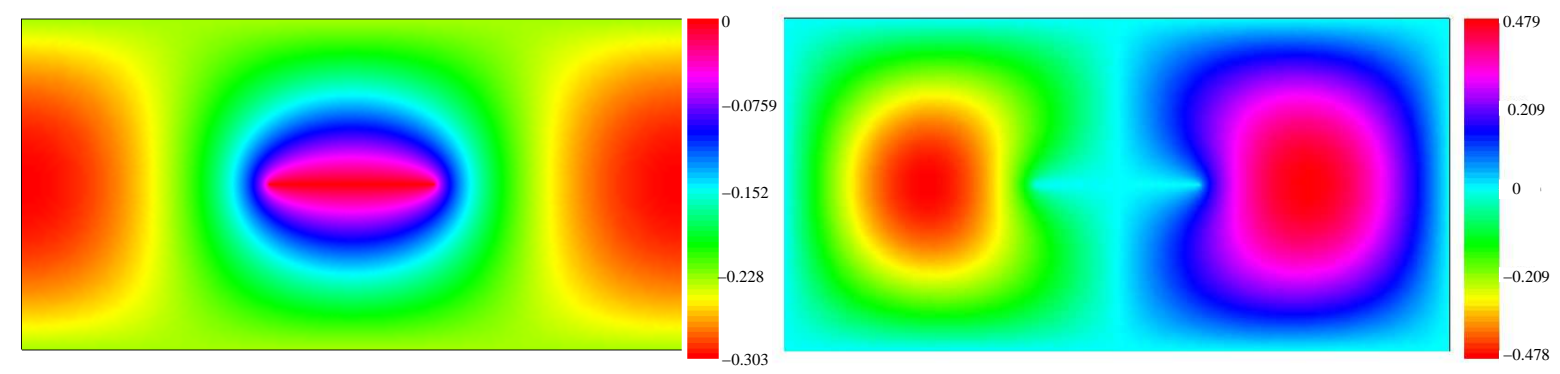

Figure 4: First two symmetrical Dirichlet eigenfunctions

\begin{tabular}{|c|c|c|c|c|c|}
\hline$\nu_{1}^{d}$ & $\nu_{2}^{d}$ & $\nu_{3}^{d}$ & $\nu_{4}^{d}$ & $\nu_{5}^{d}$ & $\nu_{6}^{d}$ \\
\hline 0.7967 & 1.6314 & 1.9266 & 2.5396 & 2.7877 & 2.9810 \\
\hline
\end{tabular}

Table 2: First six eigenvalues of the Dirichlet problem 


\subsection{The Neumann case}

A similar method has been carried out with a domain $\Omega^{n}$ described in Figure 5 . The difference between $\Omega^{n}$ and $\Omega^{d}$ comes from the fact that in the Neumann case we cannot use an exact segment to represent $S$. In order to solve this problem, the segment has been modelled as a "hole", as shown in Figure 5. The value $d$ is chosen to be very small (we found that $d=0.00005$ gives converged results) so that the "hole" approximates the segment, and we may have different values of $v^{n}$ on each side of it.

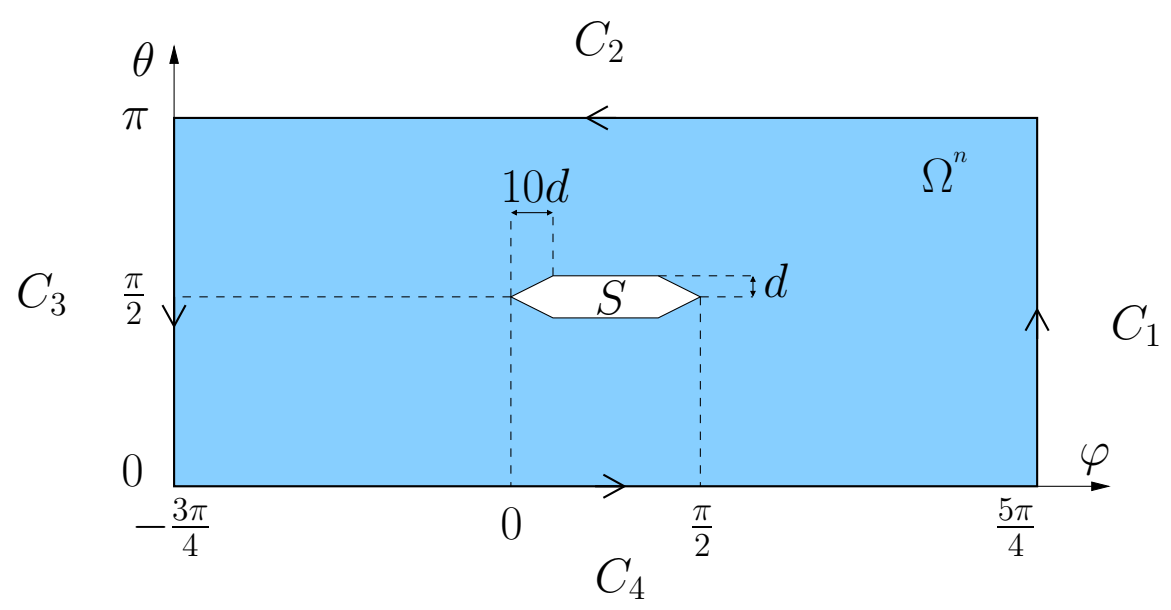

Figure 5: Domain of integration $\Omega^{n}$ in the Neumann case

Once again, the boundary conditions need to be chosen carefully. Neumann boundary conditions are used on $S$, periodic boundary conditions are imposed on $C_{1}$ and $C_{3}$, and this time anti-periodic boundary conditions need to be imposed ${ }^{1}$ on $C_{2}$ and $C_{4}$. Figure 6 shows the plot of the first two modes found by the solver and Table 3 gives the results for the first six eigenvalues obtained with a precision of order $10^{-4}$.
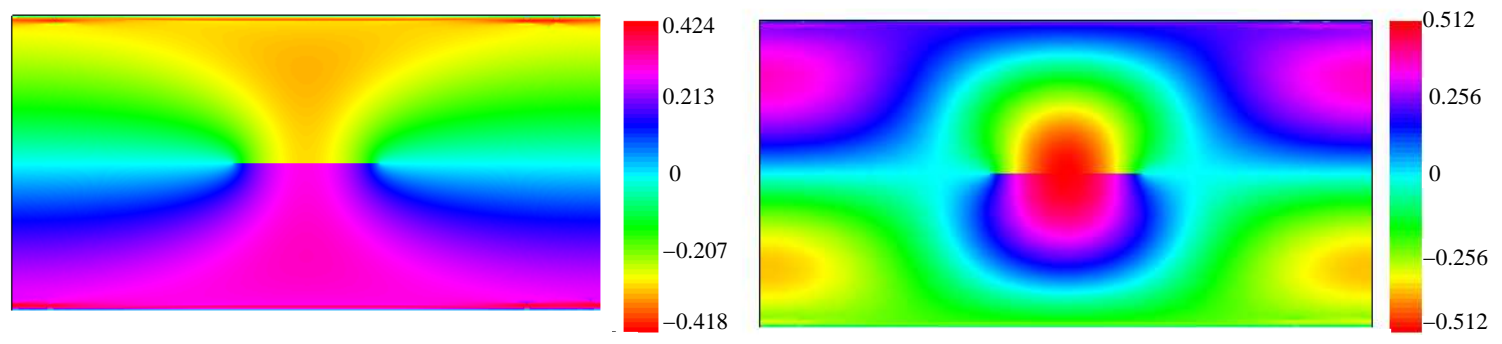

Figure 6: First two antisymmetric eigenfunctions in the Neumann case

\begin{tabular}{|c|c|c|c|c|c|}
\hline$\nu_{1}^{n}$ & $\nu_{2}^{n}$ & $\nu_{3}^{n}$ & $\nu_{4}^{n}$ & $\nu_{5}^{n}$ & $\nu_{6}^{n}$ \\
\hline 1.3145 & 2.0970 & 2.4553 & 3.0206 & 3.3011 & 3.4901 \\
\hline
\end{tabular}

Table 3: First six eigenvalues of the Neumann problem

\footnotetext{
${ }^{1} \mathrm{~A}$ simple way to impose anti-periodic boundary conditions could not be found in this case. Instead Dirichlet boundary conditions have been applied on $C_{2}$ and $C_{4}$, which explains the slight imperfections that can be observed near $C_{2}$ and $C_{4}$ in Figure 6. However, this has no effect on the value of the eigenvalues.
} 


\section{Modified Smyshlyaev formulae and spherical edge Green's functions}

Let us define the functions $\hat{v}_{1,2}^{d}(\omega, \nu, \kappa)$ to be the solutions of the Dirichlet auxiliary problem on the unit sphere with a cut $S$, with a point source located near $L_{1,2}$, with coordinates $(\kappa, \pi)$ in the local spherical coordinates and with strength $\sqrt{\frac{\pi}{\kappa}}$. The functions $\hat{v}_{1,2}^{n}(\omega, \nu, \kappa)$ are defined to be the solutions of the Neumann auxiliary problem on the unit sphere with a cut $S$, with two points source located near $L_{1,2}$, with coordinates $\left(\kappa, 0^{+}\right)$and $\left(\kappa, 0^{-}\right)$in the local spherical coordinates and with strength $\pm \frac{1}{2} \sqrt{\frac{\pi}{\kappa}}$. These two definitions correspond to the four equations

$$
\left(\tilde{\Delta}+\nu^{2}-\frac{1}{4}\right) \hat{v}_{1,2}^{d, n}(\omega, \nu, \kappa)=\sqrt{\frac{\pi}{\kappa}} \frac{\delta\left(\zeta_{1,2}-\kappa\right)}{\kappa} \mathfrak{S}_{\pi}^{d, n}\left(\phi_{1,2}\right) .
$$

As solutions of spherical auxiliary problems, the Green's function $\hat{v}_{1,2}^{d, n}(\omega, \nu, \kappa)$ must satisfy both the edge conditions and the Dirichlet/Neumann boundary conditions given in Section 2. Let us now define new functions, the spherical edge Green's functions $v_{1,2}^{d, n}(\omega, \nu)$, by

$$
v_{1,2}^{d, n}(\omega, \nu)=\lim _{\kappa \rightarrow 0} \hat{v}_{1,2}^{d, n}(\omega, \nu, \kappa) .
$$

Using Fredholm theory, and because of the symmetric/antisymmetric way in which $v_{1,2}^{d, n}$ are defined, it is possible to express them in terms of the eigenfunctions and eigenvalues computed in Section 3 by

$$
v_{1,2}^{d, n}(\omega, \nu)=2 \sum_{j} \frac{A_{1,2}^{d, n}(j) \phi_{j}^{d, n}(\omega)}{\nu^{2}-\left(\nu_{j}^{d, n}\right)^{2}},
$$

where $A_{1,2}^{d, n}(j)$ are unknown constants. This is one of the reasons why we only considered the symmetric/antisymmetric eigenfunctions in Section 3. The spherical edge Green's functions still satisfy the Dirichlet/Neumann boundary conditions, but they violate one of the edge conditions $\left(v_{1}^{d, n}\right.$ violate the edge condition on $L_{1}$ and $v_{2}^{d, n}$ that on $L_{2}$ ). The fact that one of the edge conditions is respected can be expressed mathematically by introducing the unknown coefficients $C_{12}^{d, n}(\nu)$ and $C_{21}^{d, n}(\nu)$ as

$$
\begin{aligned}
& v_{1}^{d, n}\left(\zeta_{2}, \phi_{2}, \nu\right)=\frac{2 C_{12}^{d, n}(\nu)}{\sqrt{\pi}} \zeta_{2}^{1 / 2} \mathfrak{T}^{d, n}\left(\frac{\phi_{2}}{2}\right)+O\left(\zeta_{2}^{3 / 2}\right) \\
& v_{2}^{d, n}\left(\zeta_{1}, \phi_{1}, \nu\right)=\frac{2 C_{21}^{d, n}(\nu)}{\sqrt{\pi}} \zeta_{1}^{1 / 2} \mathfrak{T}^{d, n}\left(\frac{\phi_{1}}{2}\right)+O\left(\zeta_{1}^{3 / 2}\right) .
\end{aligned}
$$

Using these spherical edge Green's functions, the following formulae (modified Smyshlyaev formulae) can be derived:

$$
\begin{aligned}
f^{d, n}\left(\omega, \omega_{0}\right) & =\frac{\mathfrak{d}^{d, n}}{4 \pi i\left(\eta+\eta_{0}\right)} \int_{\gamma} e^{-i \pi \nu}\left[v_{1}^{d, n}\left(\omega_{0}, \nu\right) v_{1}^{d, n}(\omega, \nu+1)+v_{1}^{d, n}(\omega, \nu) v_{1}^{d, n}\left(\omega_{0}, \nu+1\right)\right] d \nu \\
f^{d, n}\left(\omega, \omega_{0}\right) & =\frac{\mathfrak{d}^{d, n}}{4 \pi i\left(\xi+\xi_{0}\right)} \int_{\gamma} e^{-i \pi \nu}\left[v_{2}^{d, n}\left(\omega_{0}, \nu\right) v_{2}^{d, n}(\omega, \nu+1)+v_{2}^{d, n}(\omega, \nu) v_{2}^{d, n}\left(\omega_{0}, \nu+1\right)\right] d \nu \\
f^{d, n}\left(\omega, \omega_{0}\right) & =\frac{i}{8 \pi\left(\xi+\xi_{0}\right)\left(\eta+\eta_{0}\right)} \int_{\Gamma^{d, n}} \frac{e^{-i \pi \nu}}{\nu} C_{12}^{d, n}(\nu)\left[B^{d, n}\left(\omega, \omega_{0}, \nu\right)+B^{d, n}\left(\omega_{0}, \omega, \nu\right)\right] d \nu,
\end{aligned}
$$

where

$$
B^{d, n}\left(\omega, \omega_{0}, \nu\right)=\left(v_{1}^{d, n}(\omega, \nu+1)-v_{1}^{d, n}(\omega, \nu-1)\right)\left(v_{2}^{d, n}\left(\omega_{0}, \nu+1\right)-v_{2}^{d, n}\left(\omega_{0}, \nu-1\right)\right)
$$

and $\gamma$ and $\Gamma^{d, n}$ are the contours shown in Figure 7. 


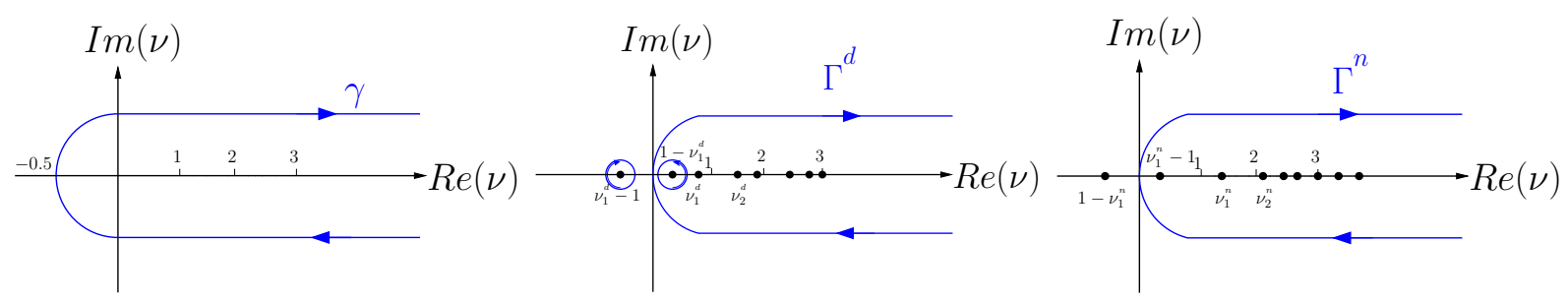

Figure 7: Contours $\gamma$ and $\Gamma^{d, n}$

Note that the difference between the contours $\Gamma^{d}$ and $\Gamma^{n}$ arises from the first eigenvalue; since $\nu_{1}^{d}<1$ while $\nu_{1}^{n}>1$, it follows that the pole at $\nu=1-\nu_{1}^{n}$ does not need to be excluded from the contour $\Gamma^{n}$, while the pole at $\nu=1-\nu_{1}^{d}$ must be excluded from $\Gamma^{d}$. The proof of the Neumann modified Smyshlyaev formulae is very similar to the proof of the Dirichlet case sketched in [13]. However, the detailed proof for both cases requires a multitude of details, and will be presented in [30]. In a recent publication [18], Shanin et al. derive some similar formulae for an arbitrary corner angle in the Dirichlet case, but for definiteness we will here consider only the quarter-plane. In order to compute the diffraction coefficient, it is essential to be able to compute the spherical edge Green's functions in an efficient way. A method fulfilling this requirement has been developed by Shanin [14] and has been implemented in the present paper. Using the following asymptotic behaviour of the spherical edge Green's functions near $L_{1,2}$,

$$
v_{i}^{d, n}\left(\phi_{j}, \zeta_{j}\right)=-\frac{\delta_{i, j}}{\sqrt{\pi}} \zeta_{j}^{-1 / 2} \mathfrak{T}^{d, n}\left(\frac{\phi_{j}}{2}\right)+\frac{2}{\sqrt{\pi}} C_{i j}^{d, n} \zeta_{j}^{1 / 2} \mathfrak{T}^{d, n}\left(\frac{\phi_{j}}{2}\right)+O\left(\zeta_{j}^{3 / 2}\right),
$$

and the symmetries of the problem ${ }^{2}$, Shanin derived a system of eight linear equations ${ }^{3}$ linking $v_{1,2}^{d, n}$ and their partial derivatives with respect to $\theta$ and $\phi$. Writing this system in matrix form leads to the coordinate equations

$$
\frac{\partial \boldsymbol{U}}{\partial \theta}=\boldsymbol{X} \boldsymbol{U} \quad, \quad \frac{\partial \boldsymbol{U}}{\partial \varphi}=\boldsymbol{Y} \boldsymbol{U}
$$

where $\boldsymbol{U}=\left(v_{1}^{d}, v_{2}^{d}, v_{1}^{n}, v_{2}^{n}\right)^{\boldsymbol{T}}$ and $\boldsymbol{X}=\left(\boldsymbol{X}_{j}^{i}\right)$ and $\boldsymbol{Y}=\left(\boldsymbol{Y}_{j}^{i}\right)$ are $4 \times 4$ matrices $^{4}$ depending on $\theta, \varphi$ and $C_{i j}^{d, n}$. Using the fact that $\boldsymbol{U}$ should satisfy the Laplace-Beltrami equation almost everywhere on the unit sphere, and using (17), one may prove that for $\nu$ not belonging to the spectrum of (5)

$$
\left(C_{11}^{d}+C_{11}^{n}\right)^{2}=\left(C_{12}^{d}\right)^{2}+\left(C_{12}^{n}\right)^{2}-\nu^{2}
$$

The result (18) is important, because it means that in $\mathbf{X}$ and $\mathbf{Y}$ there are only two unknown variables that depend on $\nu$ i.e. $C_{12}^{d}(\nu)$ and $C_{12}^{n}(\nu)$. This is due to the fact that the matrices $\boldsymbol{X}$ and $\boldsymbol{Y}$ involve $C_{11}^{d}$ and $C_{11}^{n}$ only via their sum $\left(C_{11}^{d}+C_{11}^{n}\right)$. However, only the value of $\left(C_{11}^{d}+C_{11}^{n}\right)^{2}$ is known, which means that we will have to take the square root of a complex number. This may lead to some stability problems as we will see in subsection 5.3. Note that the coordinate equation has four singular points on the sphere, in the sense that

\footnotetext{
${ }^{2}$ Note that (16) also defines the new constants $C_{11}^{d, n}$ and $C_{22}^{d, n}$, and that by symmetry, $C_{11}^{d, n}=C_{22}^{d, n}$ and $C_{12}^{d, n}=C_{21}^{d, n}$.

${ }^{3}$ In [14], these equations are written using the differential operators $T_{1,2,3}$ defined by $T_{1}=\frac{\partial}{\partial \phi_{1}}, T_{2}=\frac{\partial}{\partial \phi_{2}}$ and $T_{3}=$ $\frac{\partial}{\partial \varphi}$. At this stage, it seems that a typographical error occurred in [14]. Indeed Shanin's equation (3.9) should actually be $T_{1}\left[v^{2}\right]+T_{3}\left[w^{2}\right]=-E_{1}^{2} w^{1}+\left(C_{2}^{2}+E_{2}^{2}\right) w^{2}$ in the notations of [14], which, with the notations used in this paper, can be written as $T_{1}\left[v_{2}^{d}\right]+T_{3}\left[v_{2}^{n}\right]=-C_{21}^{n} v_{1}^{n}+\left(C_{22}^{d}+C_{22}^{n}\right) v_{2}^{n}$.

${ }^{4}$ Explicit expressions for these matrices are given in the appendix of [14], but note a typographical error in the expression for $\boldsymbol{X}_{3}^{1}: \boldsymbol{X}_{3}^{1}$ should be $\frac{2\left(C_{11}^{d}+C_{11}^{n}\right) \cos \varphi \cos ^{2} \theta-\sin \varphi}{2\left(1-\cos ^{2} \varphi \sin ^{2} \theta\right)}$.
} 
at these points the matrices $\boldsymbol{X}$ or $\mathbf{Y}$ are not well defined. Let us denote these four points $L_{1}, L_{2}, L_{3}$ and $L_{4}$. These points separate the equator into four arcs, one of them being our cut $\mathrm{S}$ (between $L_{1}$ and $L_{2}$ ). Let us also consider one point per arc, located in the middle of them (see Figure 9). Let us call those points $P_{1}, P_{2}, P_{3}$ and $P_{4}$. Finally, let us denote the North pole of the unit sphere as $A$. For a given $\nu$, a gradient algorithm to find the constants $C_{12}^{d, n}$ has been described by Shanin [14]. The idea of the algorithm is to start with a guessed value of $C_{12}^{d, n}$ and an initial condition given at $P_{1}$ by $\boldsymbol{U}\left(P_{1}\right)=(0,0,-1,1)^{\boldsymbol{T}}$. These conditions entirely define the coordinate equation that can be solved along arcs of constant $\varphi$ using a Runge-Kutta method. The difference between the known behaviour of $v_{1,2}^{n}$ at $P_{2}$ and $P_{3}$ (they should be zero there) and the behaviour of the two Neumann components of the solution at $P_{2}$ and $P_{3}$ is then used to adjust the initial guess for $C_{12}^{d, n}$. The process continues until the Neumann part of the solution is equal to zero at $P_{2}$ and $P_{3}$. The next step is to determine the exact initial value of $\boldsymbol{U}$, which should be $\boldsymbol{U}\left(P_{1}\right)=(0,0,-N, N)^{T}$. Hence we need to determine $N$. The $\operatorname{arc}\left(L_{1} A\right)$ is characterised by $\phi_{1}=\frac{\pi}{2}$. Therefore, using (16), we have the following asymptotic behaviour along this arc:

$$
v_{1}^{d}\left(\phi_{1}, \zeta_{1}\right)+v_{1}^{n}\left(\phi_{1}, \zeta_{1}\right)=-\sqrt{\frac{2}{\pi}} \zeta^{-1 / 2}+\sqrt{\frac{2}{\pi}}\left(C_{11}^{d}+C_{11}^{n}\right) \zeta^{1 / 2}+O\left(\zeta_{1}^{3 / 2}\right) \text { as } \zeta_{1} \rightarrow 0 .
$$

Let us denote the solution of the coordinate equation (entirely determined now that we know the constants) with initial boundary condition $\mathfrak{U}\left(P_{1}\right)=(0,0,-1,1)^{T}$ as $\mathfrak{U}(\theta, \varphi)=\left(\mathfrak{U}_{1}, \mathfrak{U}_{2}, \mathfrak{U}_{3}, \mathfrak{U}_{4}\right)^{T}$. Let us now solve numerically the coordinate equation along the $\operatorname{arc}\left(P_{1} A\right)$ with $\varphi$ being kept equal to $\frac{\pi}{4}$ (hence we are solving an ODE in $\theta)$ to obtain the value $\mathfrak{U}(A)$. Let us now introduce a small parameter $\varepsilon$ and solve the coordinate equation along the arc $\left(\mathrm{AL}_{1}\right)$, with $\varphi$ being kept equal to 0 , using $\mathfrak{U}(A)$ as the initial condition and stopping at a distance $\varepsilon$ from $L_{1}$, to obtain $\mathfrak{U}\left(\frac{\pi}{2}-\varepsilon, 0\right)$. Note now that, by linearity, we have $\boldsymbol{U}=N \mathfrak{U}$. Hence (19) leads to

$$
N=\frac{-\sqrt{\frac{2}{\pi}} \varepsilon^{-1 / 2}+\sqrt{\frac{2}{\pi}}\left(C_{11}^{d}+C_{11}^{n}\right) \varepsilon^{1 / 2}}{\mathfrak{U}_{1}\left(\frac{\pi}{2}-\varepsilon, 0\right)+\mathfrak{U}_{3}\left(\frac{\pi}{2}-\varepsilon, 0\right)}+O\left(\varepsilon^{3 / 2}\right) .
$$

This result can be seen graphically using Figure 8. At this stage we know all the constants of the problem (namely $C_{12}^{n}, C_{12}^{d}$ and $N$ ). Hence it is possible, by solving the coordinate equation at constant $\varphi$, for example along the arc $\left(P_{1} A\right)$, to obtain the exact value of $\boldsymbol{U}$ at the point $A$. Thereafter the process is quite simple; we tabulate the value of $\varphi$ between 0 and $2 \pi$, and for each value of $\varphi$ the coordinate equation is solved along the associated arc from $A$ to the equator, as can be seen in Figure 9. Figure 10 shows some of the spherical edge Green's functions for a particular value of $\nu$, and illustrates the fact that they violate one of the edge conditions and that they respect the Dirichlet (left plot) and Neumann (right plot) boundary conditions. Indeed, on the left plot, we can see that the function is singular at the point $(\theta, \varphi)=\left(\frac{\pi}{2}, 0\right)=L_{1}$ and that for $\theta=\frac{\pi}{2}$ and $\varphi \in\left(0, \frac{\pi}{2}\right)$, the function is equal to zero as expected from the Dirichlet boundary condition. Similarly, on the right plot, we can see that the function is singular for $(\theta, \varphi)=\left(\frac{\pi}{2}, \frac{\pi}{2}\right)=L_{2}$ and that for $\theta=\frac{\pi}{2}$ and $\varphi \in\left(\frac{\pi}{2}, 2 \pi\right)$, the function is equal to zero as expected from the Neumann boundary condition. 


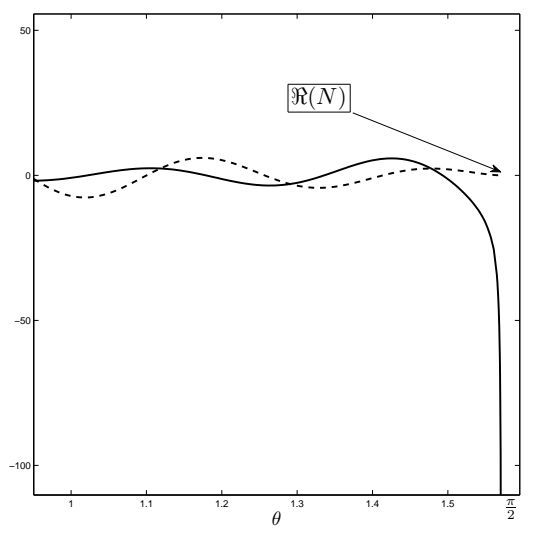

Figure 8: Illustration of the method to find $\operatorname{Re}(N)$ for $\nu=20+i$, plot (solid) of $\operatorname{Re}\left(\mathfrak{U}_{1}(\theta, 0)+\mathfrak{U}_{3}(\theta, 0)\right)$ and plot (dash) of $\operatorname{Re}\left(\frac{-\sqrt{\frac{2}{\pi}}\left(\frac{\pi}{2}-\theta\right)^{-1 / 2}+\sqrt{\frac{2}{\pi}}\left(C_{11}^{d}+C_{11}^{n}\right)\left(\frac{\pi}{2}-\theta\right)^{1 / 2}}{\mathfrak{U}_{1}(\theta, 0)+\mathfrak{U}_{3}(\theta, 0)}\right)$

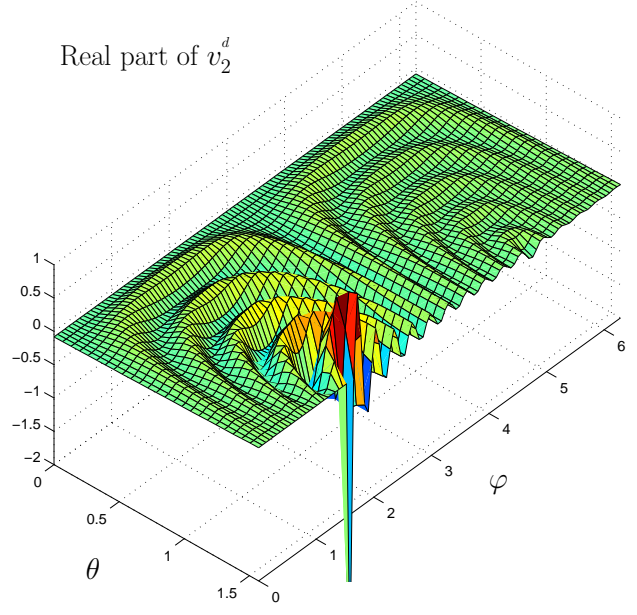

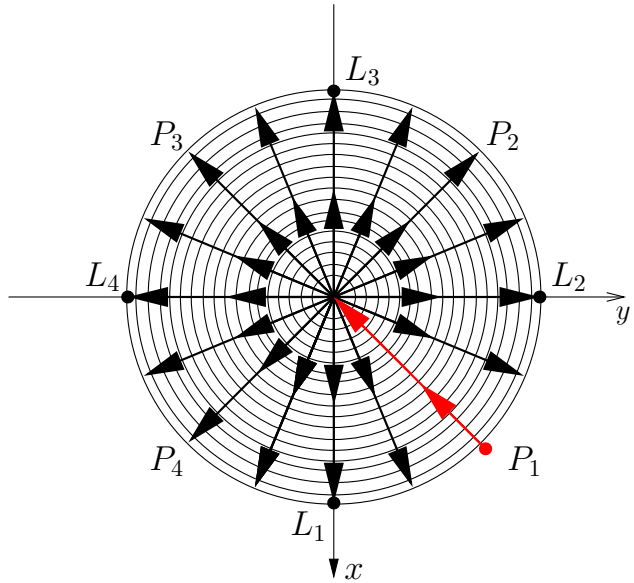

Figure 9: Illustration of the method for solving the coordinate equation, starting at $P_{1}$, going to the North pole, and going back to the equator

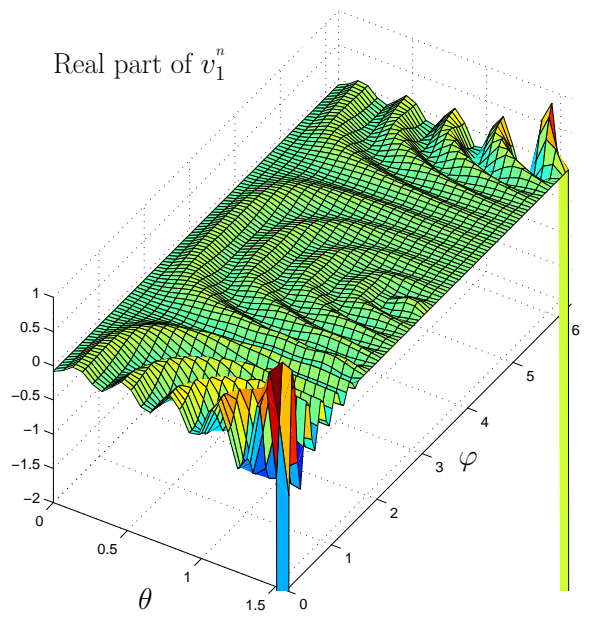

Figure 10: Real part of the second Dirichlet edge Green's function (left) and of the first Neumann edge Green's function (right) for $\nu=20+i$ 


\section{Numerical computation of $f^{d, n}\left(\omega, \omega_{0}\right)$}

\subsection{Deformation of the integration contour}

Following the work of Kamotski [31] and Babich et al [11], it can be proved that, up to a multiplicative rational function of $\nu$,

$$
\begin{aligned}
v_{1}^{d, n}(\omega, \nu) & \sim \exp \{-|\operatorname{Im}(\nu)| \arccos (\xi)\} \\
v_{2}^{d, n}(\omega, \nu) & \sim \exp \{-|\operatorname{Im}(\nu)| \arccos (\eta)\} \\
C_{12}^{d, n}(\nu) & \sim \exp \left\{-|\operatorname{Im}(\nu)| \frac{\pi}{2}\right\}
\end{aligned}
$$

as $\nu \rightarrow \infty$. Therefore, for $\arccos (\xi)+\arccos \left(\xi_{0}\right)>\pi$ (respectively $\left.\arccos (\eta)+\arccos \left(\eta_{0}\right)>\pi\right)$, we can deform the contour $\gamma$ into a contour $\gamma_{\text {new }}$ as shown on Figure 11, on which the integrand of (12) ((13) respectively) will be exponentially small at infinity. These ranges constitute the oasis zone. Figure 12 shows the oasis zone for the first modified Smyshlyaev formula, for an incident wave defined by $\omega_{0}=\left(\theta_{0}, \varphi_{0}\right)=\left(\frac{\pi}{3}, \frac{\pi}{3}\right)$. Note that the boundary of the first oasis zone corresponds to the intersection between the unit sphere and the cone radiated by the edge $\Lambda_{1}$, the apex of which is located at the quarter-plane vertex.

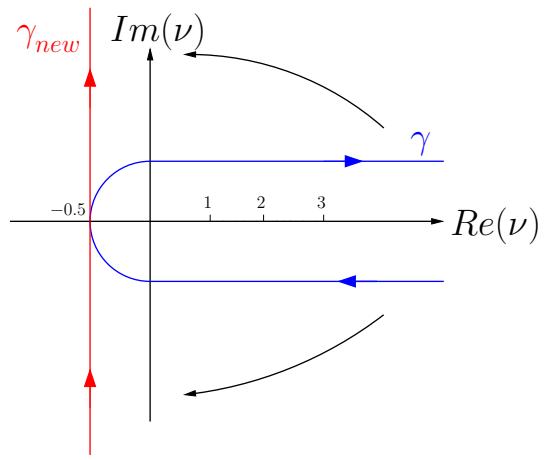

Figure 11: Illustration of the deformation of the contour $\gamma$

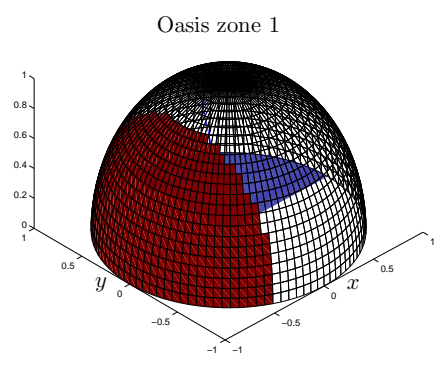

Figure 12: The oasis zone for the first formula (12) (shaded part of the unit sphere) for $\omega_{0}=\left(\theta_{0}, \varphi_{0}\right)=\left(\frac{\pi}{3}, \frac{\pi}{3}\right)$ and the quarter-plane (shaded part of the equatorial plane)

We now wish to consider the asymptotic behaviour of the integrand in the third modified Smyshlyaev formula (14). Using (20),(21), (22) and (15), it can be proved that, for $\left[\arccos \left(\xi_{0}\right)+\arccos (\eta)>\frac{\pi}{2}\right.$ and $\left.\left.\arccos (\xi)+\arccos \left(\eta_{0}\right)>\frac{\pi}{2}\right)\right]$, we can deform the contours $\Gamma^{d, n}$ into the contours $\Gamma_{\text {new }}^{d, n}$ as shown in Figure 13, on which the integrand in (14) will be exponentially small at infinity.
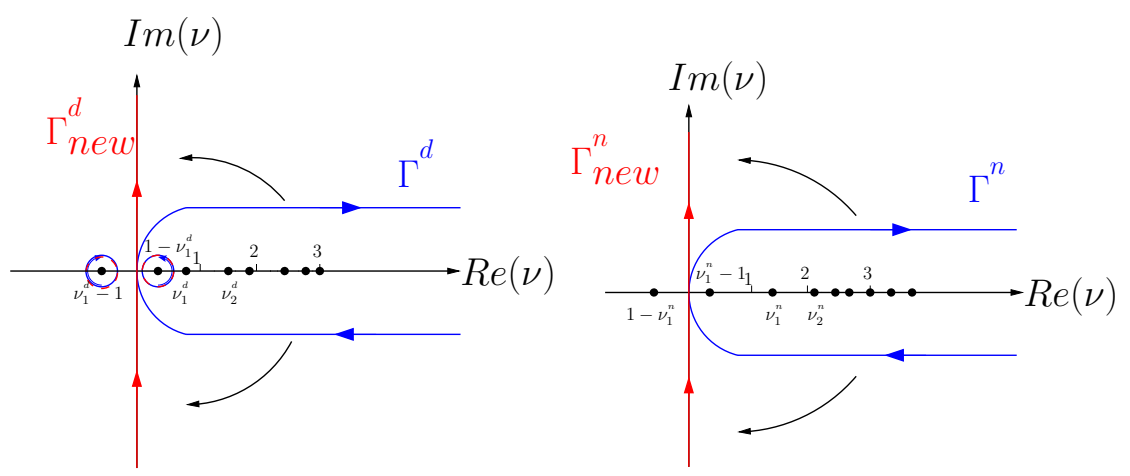

Figure 13: Illustration of the deformation of the contours $\Gamma^{d, n}$ 
Figure 14 illustrates the oasis zone 3 associated with the third Smyshlyaev formula, for the same value of $\omega_{0}$ as in Figure 12. Therefore it can be seen in this case that the third modified Smyshlyaev formula covers a much greater area of the unit sphere than the first and second formulae. Indeed the area covered by the first two formulae is included within that covered by the third formula. Note that this time, the boundary of the oasis zone 3 corresponds to the intersection between the unit sphere and the two secondary radiated cones (a secondary radiated cone corresponds to the re-scattering of a cone emanating from the other edge).
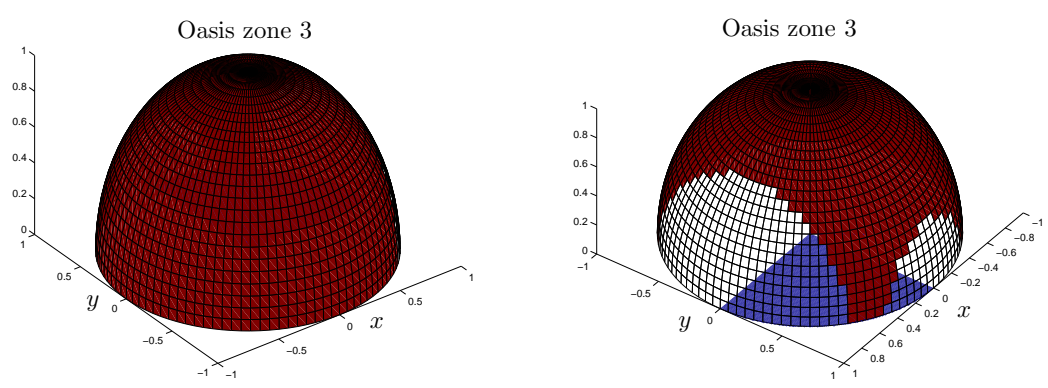

Figure 14: Two views of the oasis zone (shaded part of the unit sphere) of the third formula for $\omega_{0}=\left(\theta_{0}, \varphi_{0}\right)=\left(\frac{\pi}{3}, \frac{\pi}{3}\right)$ and the quarter-plane (shaded part of the equatorial plane)

\subsection{Useful symmetrical properties}

As demonstrated in section 4, it is possible to compute the spherical edge Green's functions and thus the integrands of the modified Smyshlyaev formulae for a given value of $\nu$ not belonging to the spectrum of (5). In order to compute the diffraction coefficient, it is necessary to perform this computation all along a contour in the $\nu$ complex plane. But let us focus first on the case of one particular $\nu$ to obtain a better understanding of the problem and derive useful properties that will significantly speed up the computation of the diffraction coefficient. The problem of finding the edge Green's functions, denoted $P(\nu)$, can be formulated as follows:

Problem 1 Let $\nu$ not belong to the spectrum of (5), we want to find constants $C_{12}^{d}(\nu), C_{12}^{n}(\nu), C_{11}^{d}(\nu)+C_{11}^{n}(\nu)$ and functions $U_{\nu}(\theta, \varphi)$ such that:

$$
\text { Problem } P(\nu):\left\{\begin{array}{l}
\left(C_{12}^{d}(\nu)\right)^{2}+\left(C_{12}^{n}(\nu)\right)^{2}-\nu^{2}=\left(C_{11}^{d}(\nu)+C_{11}^{n}(\nu)\right)^{2} \\
\frac{\partial U_{\nu}}{\partial \theta}=\left(C_{12}^{d}(\nu) \boldsymbol{L}_{1}+C_{12}^{n}(\nu) \boldsymbol{L}_{2}+\left(C_{11}^{d}(\nu)+C_{11}^{n}(\nu)\right) \boldsymbol{L}_{3}+\boldsymbol{L}_{4}\right) U_{\nu} \\
\frac{\partial U_{\nu}}{\partial \varphi}=\left(C_{12}^{d}(\nu) \boldsymbol{M}_{1}+C_{12}^{n}(\nu) \boldsymbol{M}_{2}+\left(C_{11}^{d}(\nu)+C_{11}^{n}(\nu)\right) \boldsymbol{M}_{3}+\boldsymbol{M}_{4}\right) U_{\nu} \\
\left(\begin{array}{c}
0 \\
0 \\
-1 \\
1
\end{array}\right), U_{\nu}\left(\frac{\pi}{2}, \frac{3 \pi}{4}\right)=\left(\begin{array}{c}
* \\
* \\
0 \\
* \\
* \\
* \\
*
\end{array}\right), U_{\nu}\left(\frac{\pi}{2}, \frac{5 \pi}{4}\right)=\left(\begin{array}{c}
* \\
*
\end{array}\right)
\end{array}\right.
$$

where $\boldsymbol{L}_{\boldsymbol{i}}$ and $\boldsymbol{M}_{\boldsymbol{i}}$ are 4 x4 real matrices, which can be determined from (17).

By simply taking the complex conjugate of (23), the following proposition is obtained:

Proposition 1 If $\left(C_{12}^{d}(\nu), C_{12}^{n}(\nu), C_{11}^{d}(\nu)+C_{11}^{n}(\nu), U_{\nu}\right)$ is a solution of $P(\nu)$, then $\left(\overline{C_{12}^{d}(\nu)}, \overline{C_{12}^{n}(\nu)}, \overline{C_{11}^{d}(\nu)+C_{11}^{n}(\nu)}, \overline{U_{\nu}}\right)$ is a solution of $P(\bar{\nu})$. 
Now, by replacing $\nu$ by $-\nu$ in (23), the following proposition is obtained:

Proposition 2 If $\left(C_{12}^{d}(\nu), C_{12}^{n}(\nu), C_{11}^{d}(\nu)+C_{11}^{n}(\nu), U_{\nu}\right)$ is a solution of $P(\nu)$, then $\left(C_{12}^{d}(\nu), C_{12}^{n}(\nu), C_{11}^{d}(\nu)+C_{11}^{n}(\nu), U_{\nu}\right)$ is a solution of $P(-\nu)$.

Then using the Propositions 1 and 2, we obtain the following Corollary:

Corollary 1 If $\left(C_{12}^{d}(\nu), C_{12}^{n}(\nu), C_{11}^{d}(\nu)+C_{11}^{n}(\nu), U_{\nu}\right)$ is a solution of $P(\nu)$, then $\left(\overline{C_{12}^{d}(\nu)}, \overline{C_{12}^{n}(\nu)}, \overline{C_{11}^{d}(\nu)+C_{11}^{n}(\nu)}, \overline{U_{\nu}}\right)$ is a solution of $P(-\bar{\nu})$.

These results imply that if the constants are known on a particular contour, then they are known on its symmetric image with respect to the real and the imaginary axis. Hence, for a sufficiently symmetric contour, the number of times (23) needs to be solved can be reduced by up to a factor 4 .

\subsection{Determination of the constants along the contour}

In this subsection we will consider only the most complicated case, the third modified Smyshlyaev formula in the Dirichlet case. First, we modify the contour $\Gamma_{\text {new }}^{d}$ in order to ease the numerical evaluation of the contour integral. Let us first truncate the contour along the imaginary axis at a sufficiently large finite value to ensure convergence (in many cases, truncation at $|\nu|=7$ was found to be sufficient). In order to be able to exploit the previous symmetry properties, the circles round $\nu= \pm\left(\nu_{1}^{d}-1\right)$ are changed into rectangles. Let us denote $\Gamma_{\text {num }}^{d}$, the new contour used, as described in Figure 15.

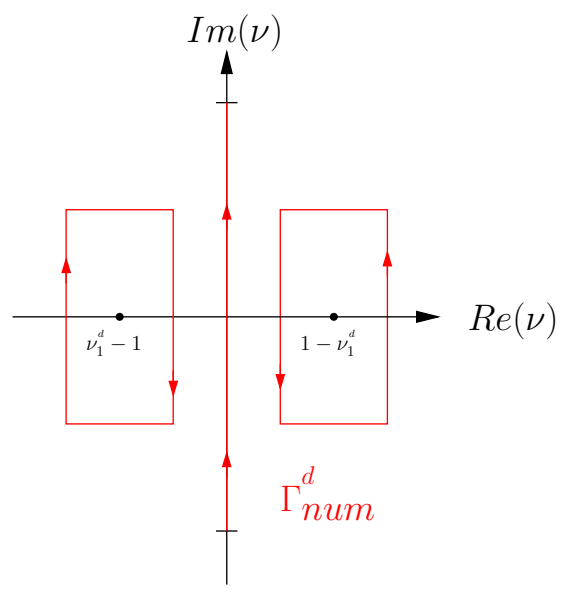

Figure 15: Contour used for numerical computation

Particular care should be taken when choosing the centre and width of the rectangles. First of all, it is necessary that $1-\nu_{1}^{d}$ and $\nu_{1}^{d}-1$ be the only eigenvalues inside the rectangles. It is equally important to ensure that none of the boundaries of the rectangles, and of their shifts by \pm 1 , encounter any of the points $\nu= \pm \nu_{j}^{d, n}$ and $\nu= \pm\left(1-\nu_{j}^{d, n}\right)$. The centres of the rectangles have been chosen to be 0.20334 and -0.20334 , with a half width of 0.05 . Figure 16 illustrates the fact that this particular choice is suitable for the computation. 


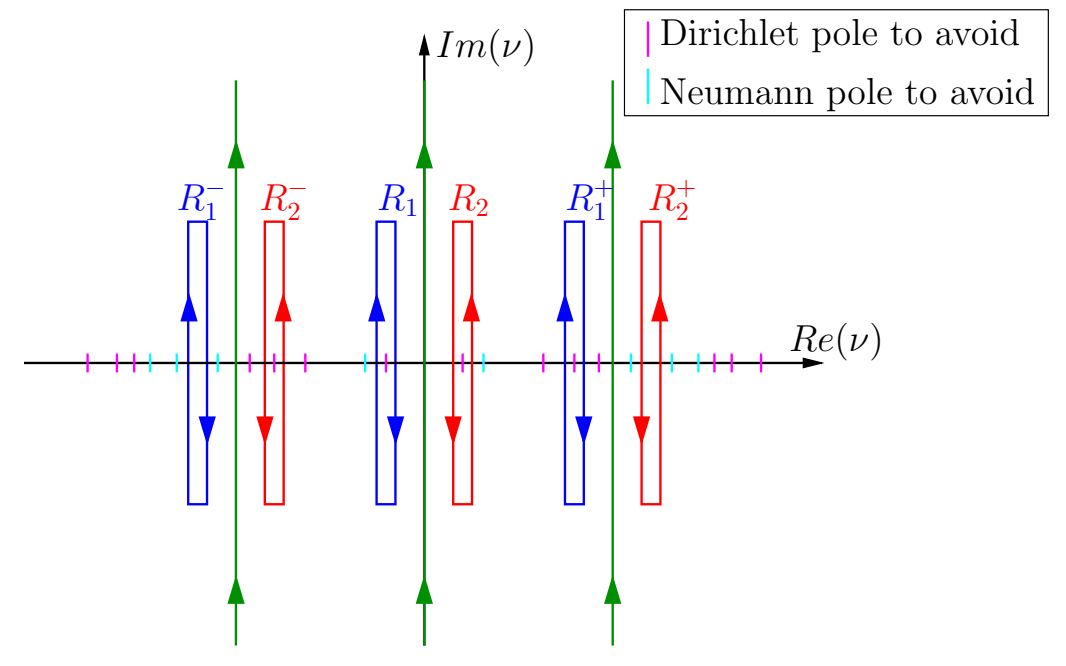

Figure 16: Vertical line, rectangles and their shifts versus the eigenvalues to avoid

Because of the form of the third embedding formula, and in particular the presence of $v_{1,2}^{d}(\nu+1), v_{1,2}^{d}(\nu-1)$ and $C_{12}^{d}(\nu)$, integrating along the contour $\Gamma_{\text {num }}^{d}$ actually implies computing the constants along each of the contours described in Figure 16. $R_{1,2}$ correspond to the rectangles of $\Gamma_{\text {num }}^{d}$, and $R_{1,2}^{+}\left(R_{1,2}^{-}\right.$respectively) correspond to their shift by +1 ( -1 respectively). This is where the properties of subsection 5.2 become very useful. Indeed, let us take any of the rectangles in Figure 16. Each vertical segment may be separated into two vertical segments, one with positive imaginary part, and the other with negative imaginary part. Proposition 1 implies that we only need to compute the constants for one of these two segments, as they are images of each other by conjugation. Similarly, the two horizontal segments of a rectangle are images of each other by conjugation and hence the constants only need to be computed on one of them. Moreover, once the constants have been computed on the rectangles $R_{1}, R_{1}^{+}$and $R_{1}^{-}$, using Corollary 1 , we automatically obtain the constants on $R_{2}, R_{2}^{+}$and $R_{2}^{-}$from

$$
R_{2}=-\overline{R_{1}}, \quad R_{2}^{+}=-\overline{R_{1}^{-}}, \quad R_{2}^{-}=-\overline{R_{1}^{+}} .
$$

So the main difficulty is to compute the constants for half of a vertical line. When $\nu$ is far from the real axis, the algorithm mentioned in Section 4 works well and converges very quickly, often in as few as three iterations. However, when we get closer to the real axis, due to the square root problem described in Section 4, the convergence becomes more difficult to achieve and the algorithm may lead to two different results, according to the sign of $\left(C_{11}^{d}+C_{11}^{n}\right)$. First, in order to accelerate the convergence, instead of taking random values of $C_{12}^{d, n}$ as the input of the algorithm it is better to use the constants computed on the previous step to initialise the algorithm. Second, for the integrals to be well defined we want the constants to be continuous functions of $\nu$. This is achieved by imposing a constant sign on the imaginary part of $\left(C_{11}^{d}+C_{11}^{n}\right)$ along the vertical half-line. Those two steps ensure fast convergence and a unique continuous output from the algorithm. The strength of the coordinate equation method is that the computation of the diffraction coefficient can be decomposed into two parts. First, as just seen, all the constants of the problem should be computed on the appropriate integration contour. This is the part requiring the most computational time ${ }^{5}$. This has only to be done once. Then, once this is done, one can obtain the value of

\footnotetext{
${ }^{5}$ In our case, 200 points have been used along each vertical lines and the running-time (using MATLAB R2010a on a modern 32-bit desktop PC) was $128 \mathrm{~s}$ for the Formula 1 and 2 together, $218 \mathrm{~s}$ for the third Formula in the Neumann case and $1125 \mathrm{~s}$ for the third Formula in the Dirichlet case.
} 
the diffraction coefficient for any incidence $\omega_{0}$ along any observing $\operatorname{arc}$ of constant $\varphi$ very quickly as can be seen in Table 4.

\section{Numerical results}

Figures 17, 19, 20 and 22 show the value of the imaginary part of the diffraction coefficient for four different incident waves (the real part of the coefficient is always zero, see Appendix B). The diffraction coefficient is computed on the Northern hemisphere of the unit sphere. For more clarity, the results are then projected onto the equatorial plane. On each plot, the boundaries of the quarter-plane are shown and the projection of the intersection between the incident wave and the unit sphere (projection of $\omega_{0}$ ) is denoted by a cross. Figure 17 shows the result only for the Dirichlet case, as in this case the incident wave direction is in the same plane as the quarter-plane so that the Neumann diffraction coefficient is zero everywhere. In [18], Shanin et al. published the value of the diffraction coefficient given by the first formula along just the dashed black line in Figure 17 (they did not give any results linked to the third formula) and didn't implement the coordinate equation method numerically (they used an integral equation instead). In all the figures the superiority of the third modified Smyshlyaev formula, and the limitations of the first two formulae, are clear. These figures also show that in some cases the third formula can cover the whole sphere (Figures 17 and 19 ), but this is not always the case as shown in Figure 20 and 22.
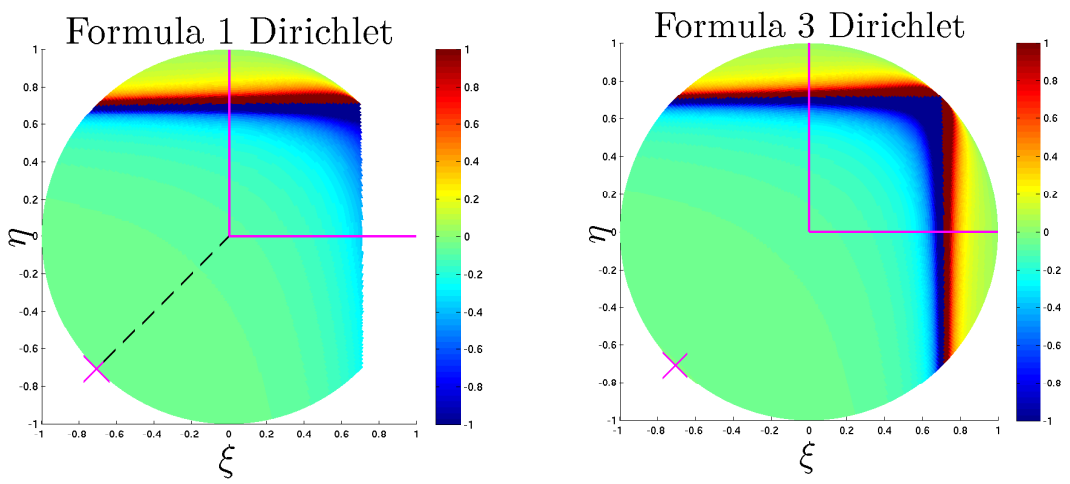

Figure 17: Imaginary part of $f^{d}$ for $\theta_{0}=\frac{\pi}{2}$ and $\varphi_{0}=\frac{5 \pi}{4}$

In order to demonstrate that these results agree with the results published in [18], Figure 18 gives the value of the Dirichlet diffraction coefficient along the black dashed line of Figure 17. Figure 18 also emphasises the perfect agreement between the first and the third Modified Smyshlyaev formulae in this case. The running time of the algorithm is also given in Table 4. 


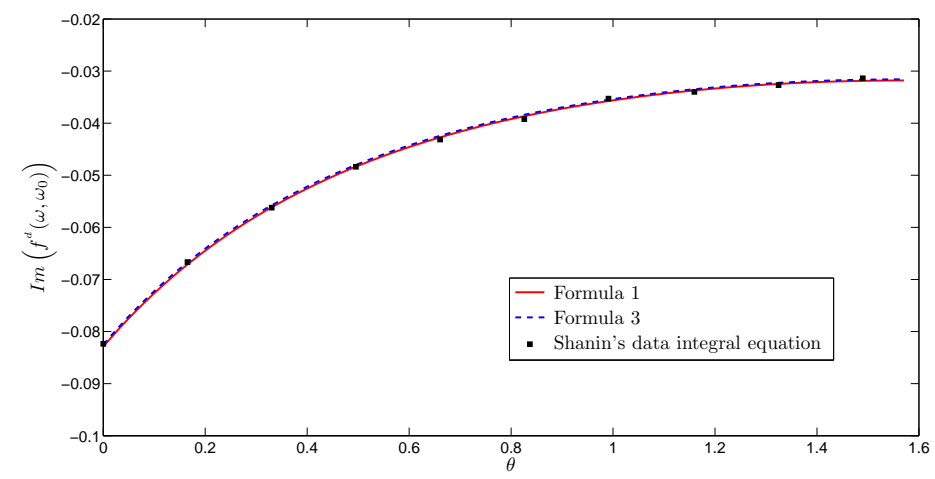

Figure 18: Imaginary part of $f^{d}$ for $\theta_{0}=\frac{\pi}{2}$ and $\varphi_{0}=\frac{5 \pi}{4}$ along the arc $\varphi=\frac{5 \pi}{4}$, comparison between results obtained by implementing the coordinate equation method for Formulae 1 and 3 and the results of [18] obtained for Formula 1 with an integral equation method

\begin{tabular}{|c|c|c|c|c|}
\hline Related Figure & Fig. 18 & Fig. 18 & Fig. 21 & Fig. 21 \\
\hline Formula & Formula 1 & Formula 3 & Formula 1 \& 2 & Formula 3 \\
\hline Time in s & 19.02 & 98.75 & 14.29 & 14.31 \\
\hline
\end{tabular}

Table 4: Computational time (using MATLAB R2010a on a modern 32-bit desktop PC) related to Figures 18 and 21, each graph being composed of 100 points
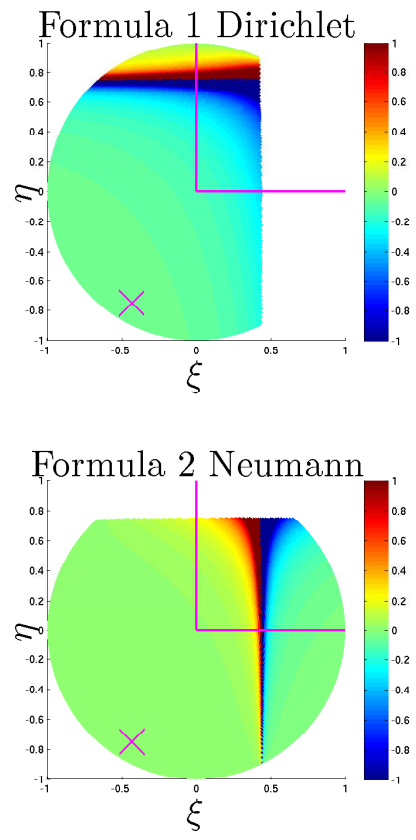
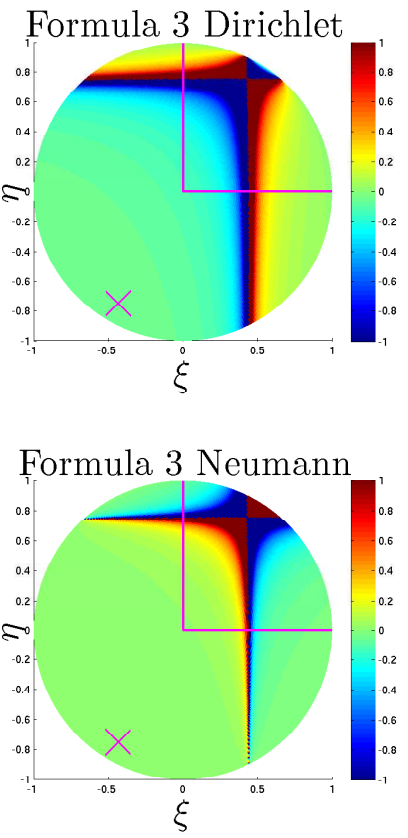

Figure 19: Imaginary part of $f^{d, n}$ for $\theta_{0}=\frac{\pi}{3}$ and $\varphi_{0}=\frac{4 \pi}{3}$ 

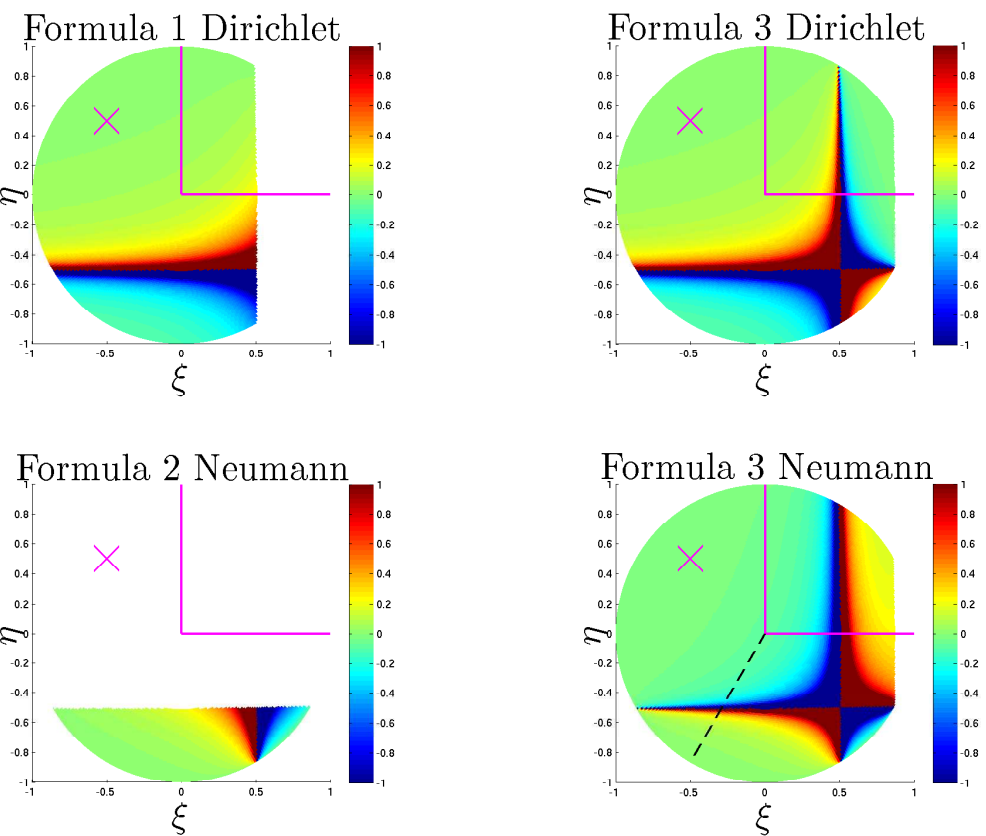

Figure 20: Imaginary part of $f^{d, n}$ for $\theta_{0}=\frac{\pi}{4}$ and $\varphi_{0}=\frac{3 \pi}{4}$

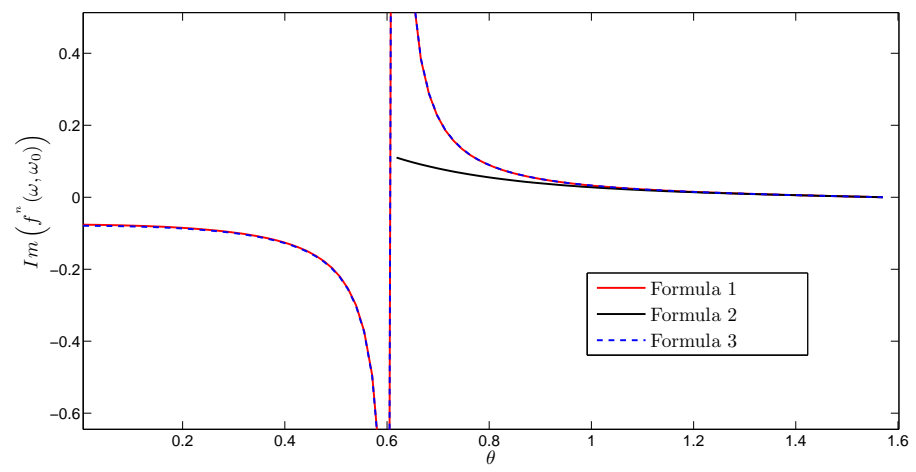

Figure 21: Imaginary part of $f^{n}$ for $\theta_{0}=\frac{\pi}{4}$ and $\varphi_{0}=\frac{3 \pi}{4}$ along the $\operatorname{arc} \varphi=\frac{4 \pi}{3}$ 

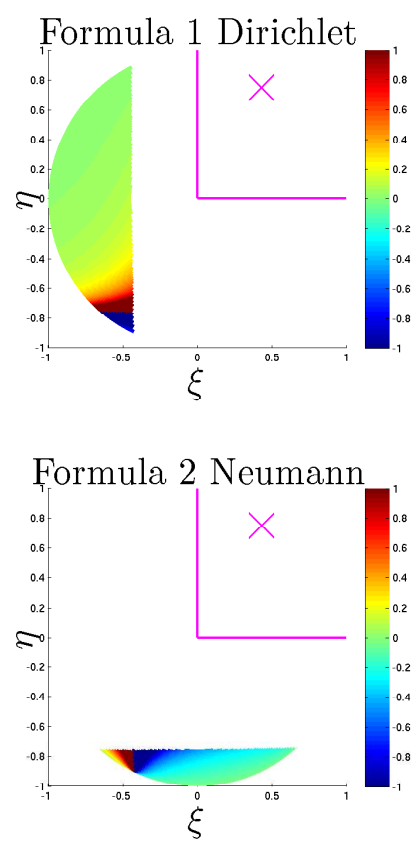
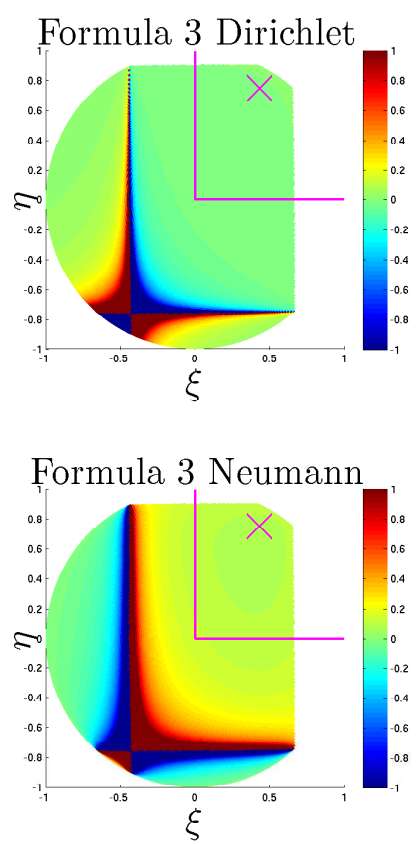

Figure 22: Imaginary part of $f^{d, n}$ for $\theta_{0}=\frac{\pi}{3}$ and $\varphi_{0}=\frac{\pi}{3}$

In all these plots we observe two singular curves $\xi=-\xi_{0}$ and $\eta=-\eta_{0}$. This is to be expected from the expressions of the modified Smyshlyaev formulae (12),(13) and (14). One of the advantages of these formulae compared to the original Smyshlyaev formula (2) is that the singularities actually appear outside the integral. Physically, these singularities correspond to the intersection between the conical waves radiated by the edges of the quarter-plane and the unit sphere, as mentioned in Section 2. The point of intersection of the singular curves corresponds to the direction of the reflected plane wave. In the area where both formula 3 and formulae 1 and 2 are defined, the results are the same, as expected. The only difference is that formulae 1 and 2 fail to describe the singularity at the boundary of their domain of definition. This is due to the fact that in the case of the two first formulae, this singularity is still "inside" the integral. In order to numerically compute these infinite integrals, we have seen that it is necessary to truncate them. This is why for the two first formulae the singularity does not appear clearly on the edge of their domain of definition. This behaviour is illustrated in Figure 21 that plots the diffraction coefficient given by different formulae along the black dashed line of Figure 20. Similarly, when not covering the entire sphere (see Figure 20 and 22), the third formula should be singular at the boundary of its domain of definition. These singularities correspond to the intersection between the secondary radiated conical waves (resulting from a primarily radiated conical wave encountering an edge) and the unit sphere.

\section{Concluding remarks}

A numerical method, based on Shanin's third modified Smyshlyaev formula and giving the three dimensional diffraction coefficient of the quarter-plane problem in the Dirichlet and the Neumann cases, has been presented. In order to do this, a method to compute the eigenvalues of the Laplace-Beltrami operator on the unit sphere with a cut has also been described. The numerical results presented here emphasise the superiority of the third modified Smyshlyaev formula, but also its limitations. Indeed, the contour deformation used for the third modified Smyshlyaev formula leads to singularities for $\arccos \left(\xi_{0}\right)+\arccos (\eta)=\frac{\pi}{2}$ 
and $\arccos \left(\eta_{0}\right)+\arccos (\xi)=\frac{\pi}{2}$. This corresponds to the singular curves

$$
\eta=\sqrt{1-\xi_{0}^{2}} \quad, \quad \xi=\sqrt{1-\eta_{0}^{2}}
$$

The first singular curve only appears ${ }^{6}$ if $\xi_{0}>0$ and the second only if $\eta_{0}>0$. This is why the domain is totally covered in the case of Figure $19\left(\xi_{0}=-\frac{\sqrt{3}}{4}, \eta_{0}=-\frac{3}{4}\right)$ and is not fully covered in the case of Figure $22\left(\xi_{0}=\frac{\sqrt{3}}{4}, \eta_{0}=\frac{3}{4}\right)$. The fact that, due to the geometry of the problem, no third conical radiated waves can occur seems to indicate that we are only one modified Smyshlyaev formula (the fourth) away from a total solution of the diffraction of acoustic waves by an ideal quarter-plane. It seems reasonable to believe that this ultimate modified Smyshlyaev formula should have a factor of the form $\left\{\left(\eta+\eta_{0}\right)\left(\xi+\xi_{0}\right)\left(\xi-\sqrt{1-\eta_{0}^{2}}\right)\left(\eta-\sqrt{1-\xi_{0}^{2}}\right)\right\}^{-1}$ in front of its integral. Further work is being carried out in order to derive this ultimate modified Smyshlyaev formula, and also to extend Shanin's theory to the case of a Robin boundary condition.

\section{Acknowledgements}

R.C. Assier acknowledges the support of the EPSRC and the Cambridge European Trust.

\footnotetext{
${ }^{6}$ Indeed, if $\xi_{0}>0$ and $\eta_{0}<0$, only one set of secondary conical waves is radiated (by $\Lambda_{2}$ ); if $\xi_{0}<0$ and $\eta_{0}>0$ (see Figure 20 ), only one set of secondary conical waves is radiated (by $\Lambda_{1}$ ); and if $\xi_{0}>0$ and $\eta_{0}>0$, two sets of secondary conical waves are radiated.
} 


\section{References}

[1] J. B. Keller, Geometrical theory of diffraction, J. Opt. Soc. Am. 52 (1962) 116-\&.

[2] L. Kraus, L. M. Levine, Diffraction by an elliptic cone, Commun. Pure Appl. Math. 14 (1961) 49-\&.

[3] R. Satterwhite, Diffraction by a quarter plane, exact solution, and some numerical results, IEEE Trans. Antennas Propag. AP22 (1974) 500-503.

[4] T. B. Hansen, Corner diffraction coefficients for the quarter plane, IEEE Trans. Antennas Propag. 39 (1991) 976-984.

[5] S. Blume, Spherical-multipole analysis of electromagnetic and acoustical scattering by a semi-infinite elliptic cone, IEEE Antennas Propag. Mag. 38 (1996) 33-44.

[6] J. Radlow, Diffraction by a quarter-plane, Arch. Ration. Mech. Anal. 8 (1961) 139-158.

[7] E. Meister, Some solved and unsolved canonical problems of diffraction theory, Lect. Notes Math. 1285 (1987) $320-336$.

[8] M. Albani, On Radlow's quarter-plane diffraction solution, Radio Sci. 42 (2007).

[9] V. P. Smyshlyaev, Diffraction by conical surfaces at high-frequencies, Wave Motion 12 (1990) 329-339.

[10] V. M. Babich, D. B. Dement'ev, B. A. Samokish, On the diffraction of high-frequency waves by a cone of arbitrary shape, Wave Motion 21 (1995) 203-207.

[11] V. M. Babich, D. B. Dement'ev, B. A. Samokish, V. P. Smyshlyaev, On evaluation of the diffraction coefficients for arbitrary "nonsingular" directions of a smooth convex cone, SIAM J. Appl. Math. 60 (2000) 536-573.

[12] B. D. Bonner, I. G. Graham, V. P. Smyshlyaev, The computation of conical diffraction coefficients in high-frequency acoustic wave scattering, SIAM J. Numer. Anal. 43 (2005) 1202-1230.

[13] A. V. Shanin, Modified Smyshlyaev's formulae for the problem of diffraction of a plane wave by an ideal quarter-plane, Wave Motion 41 (2005) 79-93.

[14] A. V. Shanin, Coordinate equations for a problem on a sphere with a cut associated with diffraction by an ideal quarterplane, Q. J. Mech. Appl. Math. 58 (2005) 289-308.

[15] M. H. Williams, Diffraction by a finite strip, Q. J. Mech. Appl. Math. 35 (1982) 103-124.

[16] R. V. Craster, A. V. Shanin, E. M. Doubravsky, Embedding formulae in diffraction theory, Proc. R. Soc. London Ser. A-Math. Phys. Eng. Sci. 459 (2003) 2475-2496.

[17] R. V. Craster, A. V. Shanin, Embedding formulae for diffraction by rational wedge and angular geometries, Proc. R. Soc. A-Math. Phys. Eng. Sci. 461 (2005) 2227-2242.

[18] E. A. Skelton, R. V. Craster, A. V. Shanin, V. Valyaev, Embedding formulae for scattering by three-dimensional structures, Wave Motion 47 (2010) 299-317.

[19] D. S. Jones, The eigenvalues of $\nabla^{2} u+\lambda u=0$ when the boundary conditions are given on semi-infinite domains, Proceedings of the Cambridge Philosophical Society 49 (1953) 668-684.

[20] W. L. Miranker, Uniqueness and representation theorems for solutions of $\Delta u+k^{2} u=0$ in infinite domains, Journal of Mathematics and Mechanics 6 (1957) 847-858.

[21] G. M. Keith, N. Peake, High-wavenumber acoustic radiation from a thin-walled axisymmetric cylinder, J. Sound Vibr. 255 (2002) 129-146.

[22] G. M. Keith, N. Peake, High-wavenumber acoustic radiation from a thin-walled scarfed cylinder, J. Sound Vibr. 255 (2002) $147-160$.

[23] A. Popov, A. Ladyzhensky (Brodskaya), S. Khozioski, Uniform asymptotics of the wave diffracted by a cone of arbitrary cross section, Russ. J. Math. Phys. 16 (2009) 296-299.

[24] V. M. Babich, On PC Ansatzs, Journal of Mathematical Sciences 132 (2006) 2-10. 10.1007/s10958-005-0470-y.

[25] B. G. Nikolaev, Wave processes in diffraction by a perfectly reflecting cone in the axisymmetric case, Journal of Mathematical Sciences 3 (1975) 125-141. 10.1007/BF01084811.

[26] J. Boersma, J. K. M. Jansen, Electromagnetic field singularities at the tip of an elliptic cone, http://alexandria.tue. nl/repository/books/357827.pdf, 1990.

[27] B. A. Hargrave, B. D. Sleeman, The numerical solution of two-parameter eigenvalue problems in ordinary differential equations with an application to the problem of diffraction by a plane angular sector, Teaching Mathematics and its Applications 14 (1994) 9-22.

[28] A. T. Abawi, R. F. Dashen, H. Levine, The eigenvalues of the laplacian on a sphere with boundary conditions specified on a segment of a great circle, J. Math. Phys. 38 (1997) 1623-1649.

[29] O. Pironneau, F. Hecht, J. Morice, freeFEM++, http://www.freefem.org, 2004.

[30] R. C. Assier, Ph.D. thesis, DAMTP, University of Cambridge, UK, 2011.

[31] V. V. Kamotski, Calculation of some integrals describing wave fields, Journal of Mathematical Sciences 108 (2002) 665-673. 10.1023/A:1013243011177.

\section{Appendix A. On the variational formulation}

In this appendix we will derive the variational formulation of the Dirichlet problem (5). Let us call $P_{2} b$ the space of continuous quadratic functions on the elements of the mesh plus bubble functions. The original $\mathrm{PDE}$ we want to solve is

$$
-\frac{1}{\sin (\theta)} \frac{\partial}{\partial \theta}\left(\sin (\theta) \frac{\partial v^{d}}{\partial \theta}\right)-\frac{1}{\sin ^{2}(\theta)} \frac{\partial^{2} v^{d}}{\partial \varphi^{2}}=\lambda v^{d} .
$$


Let $w^{d} \in P_{2} b$, if we multiply (A.1) by $w^{d}$ and then integrate it over the whole domain $\Omega^{d}$, we obtain

$$
-\int_{\Omega^{d}} \frac{\partial}{\partial \theta}\left(\sin (\theta) \frac{\partial v^{d}}{\partial \theta}\right) \frac{w^{d}}{\sin (\theta)} d \Omega^{d}-\int_{\Omega^{d}} \frac{\partial^{2} v^{d}}{\partial \varphi^{2}} \frac{w^{d}}{\sin ^{2}(\theta)} d \Omega^{d}=\lambda \int_{\Omega^{d}} v^{d} w^{d} d \Omega^{d} .
$$

At this stage, integration by parts is performed on the two terms on the RHS of (A.2) to obtain

$$
\begin{aligned}
\lambda \int_{\Omega^{d}} v^{d} w^{d} d \Omega^{d}= & \int_{\Omega^{d}}\left\{\frac{\partial v^{d}}{\partial \theta} \frac{\partial w^{d}}{\partial \theta}-\frac{\cos (\theta)}{\sin (\theta)} \frac{\partial v^{d}}{\partial \theta} w^{d}\right\} d \Omega^{d}-\int_{\partial \Omega^{d}}\left\{w^{d} \frac{\partial v^{d}}{\partial \theta}\right\} d \ell \\
& +\int_{\Omega^{d}} \frac{1}{\sin ^{2}(\theta)} \frac{\partial v^{d}}{\partial \varphi} \frac{\partial w^{d}}{\partial \varphi} d \Omega^{d}-\int_{\partial \Omega^{d}}\left\{\frac{1}{\sin ^{2}(\theta)} w^{d} \frac{\partial v^{d}}{\partial \varphi}\right\} d \ell
\end{aligned}
$$

Due to the boundary conditions, the integrals in (A.3) on the border $\partial \Omega^{d}$ are equal to zero. This leads to the final variational formulation of the problem:

$$
\forall w \epsilon P_{2} b, \int_{\Omega^{d}}\left\{\frac{\partial v^{d}}{\partial \theta} \frac{\partial w^{d}}{\partial \theta}-\frac{\cos (\theta)}{\sin (\theta)} \frac{\partial v^{d}}{\partial \theta} w^{d}+\frac{1}{\sin ^{2}(\theta)} \frac{\partial v^{d}}{\partial \varphi} \frac{\partial w^{d}}{\partial \varphi}\right\} d \Omega^{d}=\lambda \int_{\Omega^{d}} u^{d} w^{d} d \Omega^{d} .
$$

The Neumann variational formulation is very similar. The only difference is that in the Dirichlet case, the Dirichlet boundary condition has to be specified in addition to the variational formulation, while in the Neumann case, the Neumann boundary condition is automatically satisfied (natural boundary condition).

Remark 1 Note that, as mentioned in Subsection 3.1, the domain $\Omega^{d}$ used in the formulation A.4 is a rectangle, which means that $d \Omega^{d}=d \theta d \varphi$. A rectangular domain approach using periodic boundary conditions on the edges has been preferred to the standard FEM on the surface of a sphere in order to avoid certain problems with the sphere geometry. A careful reader would notice that the classic variational formulation on the sphere should differ slightly from A.4. Indeed in this case, the term in $\cot (\theta)$ should disappear. However, as expected, both methods give the same results in terms of the eigenvalues we are interested in.

\section{Appendix B. Proof that the diffraction coefficient is purely imaginary} form

In the case of the first two modified Smyshlyaev formulae (12) and (13), the integrals involved have the

$$
I_{1,2}^{d, n}=\int_{-1 / 2-i \infty}^{-1 / 2+i \infty} e^{-i \pi \nu}\left[v_{1,2}^{d, n}\left(\omega_{0}, \nu\right) v_{1,2}^{d, n}(\omega, \nu+1)+v_{1,2}^{d, n}(\omega, \nu) v_{1,2}^{d, n}\left(\omega_{0}, \nu+1\right)\right] \mathrm{d} \nu
$$

One useful fact is that if $\nu$ stays on the vertical line $(-i \infty-1 / 2, i \infty-1 / 2)$, we can write $\nu+1=-\bar{\nu}$, and using this fact together with Corollary 1 , we obtain

$$
\begin{aligned}
I_{1,2}^{d, n} & =\int_{-1 / 2-i \infty}^{-1 / 2+i \infty} e^{-i \pi \nu}\left[v_{1,2}^{d, n}\left(\omega_{0}, \nu\right) v_{1,2}^{d, n}(\omega,-\bar{\nu})+v_{1,2}^{d, n}(\omega, \nu) v_{1,2}^{d, n}\left(\omega_{0},-\bar{\nu}\right)\right] \mathrm{d} \nu \\
& =\int_{-1 / 2-i \infty}^{-1 / 2+i \infty} e^{-i \pi \nu}\left[v_{1,2}^{d, n}\left(\omega_{0}, \nu\right) \overline{v_{1,2}^{d, n}(\omega, \nu)}+v_{1,2}^{d, n}(\omega, \nu) \overline{v_{1,2}^{d, n}\left(\omega_{0}, \nu\right)}\right] \mathrm{d} \nu \\
& =-\int_{-\infty}^{+\infty} 2 e^{\pi x} \operatorname{Re}\left(v_{1,2}^{d, n}\left(\omega_{0},-\frac{1}{2}+i x\right) \overline{v_{1,2}^{d, n}\left(\omega,-\frac{1}{2}+i x\right)}\right) \mathrm{d} x
\end{aligned}
$$


Hence the integrals are real and in these cases the diffraction coefficient is purely imaginary. In the case of the third modified Smyshlyaev formula (14), the integrals involved have the forms

$$
\begin{aligned}
I_{3}^{d} & =I_{\text {line }}^{d}+I_{R_{1}}^{d}+I_{R_{2}}^{d} \\
I_{3}^{n} & =I_{\text {line }}^{n}
\end{aligned}
$$

Let us start by proving that

$$
I_{\text {line }}^{d, n}=\int_{-i \infty}^{+i \infty} \frac{e^{-i \pi \nu}}{\nu} C_{12}^{d, n}(\nu)\left[B^{d, n}\left(\omega, \omega_{0}, \nu\right)+B^{d, n}\left(\omega_{0}, \omega, \nu\right)\right] \mathrm{d} \nu
$$

is real. To prove that $B^{d, n}\left(\omega, \omega_{0}, \nu\right)$ is real on the line $(-i \infty, i \infty)$, first remark that if $\nu$ belongs to this line, then $\nu-1=-\overline{(\nu+1)}$, and apply Corollary 1 ,

$$
\begin{aligned}
& B^{d, n}\left(\omega, \omega_{0}, \nu\right)=\left(v_{1}^{d, n}(\omega, \nu+1)-v_{1}^{d, n}(\omega, \nu-1)\right)\left(v_{2}^{d, n}\left(\omega_{0}, \nu+1\right)-v_{2}^{d, n}\left(\omega_{0}, \nu-1\right)\right) \\
& =\left(v_{1}^{d, n}(\omega, \nu+1)-v_{1}^{d, n}(\omega,-\overline{(\nu+1)})\left(v_{2}^{d, n}\left(\omega_{0}, \nu+1\right)-v_{2}^{d, n}\left(\omega_{0},-\overline{(\nu+1)}\right)\right.\right. \\
& =\left(v_{1}^{d, n}(\omega, \nu+1)-\overline{v_{1}^{d, n}(\omega, \nu+1)}\right)\left(v_{2}^{d, n}\left(\omega_{0}, \nu+1\right)-\overline{v_{2}^{d, n}\left(\omega_{0}, \nu+1\right)}\right) \\
& =-4 \operatorname{Im}\left(v_{1}^{d, n}(\omega, \nu+1)\right) \operatorname{Im}\left(v_{2}^{d, n}\left(\omega_{0}, \nu+1\right)\right) \text {. }
\end{aligned}
$$

Hence $B^{d, n}\left(\omega, \omega_{0}, \nu\right)$ is real. Moreover on this same vertical line we also have $\nu=-\bar{\nu}$. Hence $C_{12}^{d, n}(\nu)=$ $C_{12}^{d, n}(-\bar{\nu})=\overline{C_{12}^{d, n}(\nu)}$, which means that $C_{12}^{d, n}(\nu)$ is real on this vertical line too. Now

$$
\begin{aligned}
I_{\text {line }}^{d, n} & =\int_{-\infty}^{\infty} \frac{e^{\pi x}}{i x} C_{12}^{d, n}(i x)\left[B^{d, n}\left(\omega, \omega_{0}, i x\right)+B^{d, n}\left(\omega_{0}, \omega, i x\right)\right] i \mathrm{~d} x \\
& =\int_{-\infty}^{\infty} \frac{e^{\pi x}}{x} C_{12}^{d, n}(i x)\left[B^{d, n}\left(\omega, \omega_{0}, i x\right)+B^{d, n}\left(\omega_{0}, \omega, i x\right)\right] \mathrm{d} x
\end{aligned}
$$

Hence $I_{\text {line }}^{d, n}$ is real, and using (14), this proves that in the Neumann case the diffraction coefficient is purely imaginary. To prove it in the Dirichlet case, we also need to prove that $I_{R_{1}}^{d}+I_{R_{2}}^{d}$ is real. Let us call $g(\nu)=$ $C_{12}^{d}(\nu)\left[B^{d}\left(\omega, \omega_{0}, \nu\right)+B^{d}\left(\omega_{0}, \omega, \nu\right)\right]$. Using the Corollary 1 , it is obvious that $g(-\bar{\nu})=\overline{g(\nu)}$. Therefore we can write

$$
\begin{aligned}
I_{R_{2}}^{d}=\int_{R_{2}} \frac{e^{-i \pi \nu}}{\nu} g(\nu) d \nu & =-\int_{R_{1}} \frac{e^{i \pi \bar{\nu}}}{-\bar{\nu}} g(-\bar{\nu}) d \nu \\
& =\overline{\int_{R_{1}} \frac{e^{-i \pi \nu}}{\nu} g(\nu) d \nu}=\overline{I_{R_{1}}^{d}},
\end{aligned}
$$

so that $I_{R_{1}}^{d}+I_{R_{2}}^{d}$ is real. Hence the diffraction coefficient in the Dirichlet case for the third modified Smyshlyaev formula is purely imaginary.

The authors believe that the diffraction coefficient being purely imaginary is a more general result and is not only due to the particular form of the modified Smyshlyaev's formulae. It may for example be due to the scale invariance of the conical shape of the scatterer, however, no proof has been found so far. This is why we decided to present a result based on the particular form of the modified Smyshlyaev's formulae. 